\title{
Perturbative Approach to the Quasinormal Modes of Dirty Black Holes
}

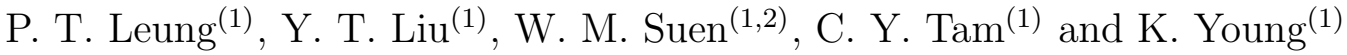 \\ (1)Department of Physics, The Chinese University of Hong Kong, Hong Kong, China \\ (2) McDonnell Center for the Space Sciences, Department of Physics, Washington University, \\ St Louis, MO 63130, US A
}

(October 8, 2018)

\begin{abstract}
Using a recently developed perturbation theory for uasinormal modes (QNM's), we evaluate the shifts in the real and imaginary parts of the QNM frequencies due to a quasi-static perturbation of the black hole spacetime. We show the perturbed QNM spectrum of a black hole can have interesting features using a simple model based on the scalar wave equation.
\end{abstract}

PACS numbers: 04.30.Db 


\section{INTRODUCTION}

The observational consequences of black holes interacting with their astrophysical environments have been a subject of much interest for the last 30 years. Within the next few years, it is expected that the new generation of gravitational wave observatories (LIGO, VIRGO) [1] will be able to detect gravitational waves emitted by black holes excited by matter, or even other black holes, falling into them. It has long been known that the gravitational waves emitted in such a process will carry a signature associated with the well-defined quasinormal mode (QNM) frequencies of the black hole [2], and will, among other things, provide confirmation of the existence of black holes. Numerical simulations [3] suggest that in some cases the QNM ringing may even dominate the signal.

QNMs of black holes have been extensively studied with the black hole perturbation theory [4. If a black hole settles down in an otherwise empty and asymptotically flat spacetime at the end-point of dynamical evolution, it will be a Kerr black hole (Schwarzschild hole in the case of zero angular momentum) [5]. Weak (linearized) gravitational waves propagating on the Kerr or Schwarzschild background can be described by the Klein-Gordon equation [4]:

$-\mathrm{kg}$

$$
\left[\partial_{t}^{2}-\partial_{x}^{2}+V(x)\right] \Phi(x, t)=0
$$

where $x$ is a radial coordinate, $\Phi$ is the radial part of a combination of the linearized changes in the metric functions representing the gravitational wave, and the outgoing wave boundary condition is appropriate for waves escaping to infinity. The potential $V(x)$ describes the scattering of the gravitational waves by the background geometry. For example, in the case of a Schwarzschild hole of mass $M, V$ is the Regge-Wheeler potential [4.6]: - $\mathrm{schw}$

$$
V(x)=\left(1-\frac{2 M}{r}\right)\left[\frac{l(l+1)}{r^{2}}+\left(1-s^{2}\right) \frac{2 M}{r^{3}}\right]
$$

for each angular momentum sector $l$, where $x=r+2 M \ln (r / 2 M-1), s$ is the spin of the field ( $s=2$ for gravitational waves), and $r$ is the circumferential radius.

A single-frequency solution $[\Phi \propto \exp (-i \omega t)]$ with the outgoing wave boundary condition is a QNM, with Im $\omega<0$. The QNM spectra of Kerr and Schwarzschild black holes have been extensively studied theoretically [7] and numerically [8,9], and the positions of the QNM frequencies in the $\omega$-plane are known in detail [8,9].

For each given $l$ in (1.2), the QNMs extend downwards in a string in the $\omega$-plane, with Re $\omega$ nearly constant and Im $\omega$ nearly uniformly spaced [8] (Fig. 1). The known pattern of frequencies provides a template against which one can try to determine the nature of the source. For an isolated black hole, the no-hair theorem [5] implies that the spectrum is described by only two parameters, the mass $M$ and the angular momentum $J$ of the hole. However, the black holes that are likely to be observed will not be isolated, but will likely be situated at the centers of galaxies, or will be surrounded by massive accretion disks. Therefore the observed spectra should not be matched against those of a pure Kerr or Schwarzschild black hole, but to one perturbed by its surrounding — a dirty black hole. We should immediately caution the reader that while gravitational waves from black holes are 
expected to be detected within the next few years, a determination of the QNM spectrum with the frequencies of a few modes included might not be an easy task. Indeed to what extent the gravitational radiation from realistic black hole events would be dominated the QNM spectrum is still a matter of much controversy. However, in as much as the goal of the gravitational wave observatories is to obtain astrophysical information of our universe (the "O" in "LIGO"), there is no doubt that we will eventually have to face this problem of the QNM spectra of dirty black holes.

We note that two kinds of perturbations are involved here. In the standard black hole perturbation theory [4], (11.1) is obtained by linearizing the metric about the Kerr or Schwarzschild background, and the time-independent eigenvalue problem (with the outgoing wave boundary condition) determines the QNM spectra of isolated holes. The second type of perturbations are the perturbations that change the background on which the wave propagates, e.g., by the presence of an accretion disk near the black hole. The perturbation of the background can often be regarded as quasi-static, and hence separable from that of the gravitational wave perturbation by the time scales involved (in a suitable gauge choice). In this paper we focus on time-independent perturbation of the background described by (1.1) with a potential $V(x)=V_{0}(x)+\mu V_{1}(x),|\mu| \ll 1$ (a model problem is given in Sect. IV below). We are led to study the QNM frequencies of the following eigenvalue problem in powers of $\mu$ :

$-\mathrm{kg} 1$

$$
-\phi^{\prime \prime}(x)+\left[V_{0}(x)+\mu V_{1}(x)\right] \phi=\omega^{2} \phi .
$$

(1.3) is appropriate for considering the perturbed Klein-Gordon wave equation describing the propagation of scalar waves in a gravitationally perturbed (dirty) black hole spacetime (as will be shown below). In this paper we show how the disturbed QNM spectrum can be determined for such a system. This represents the first step towards determining the disturbed gravitational wave QNM spectrum of a dirty hole, the real case of physical interest. For a realistic black hole perturbed by an external matter fluid source, the gravitational wave QNM spectrum also involves the fluid modes. Just as in the case of the perturbation of fluid star, we would expect two types of perturbations, with one strongly involving the fluid motion (e.g., the polar $f, g, p$ modes), while the other only weakly involving the fluid (e.g., the axial modes). We expect the scalar perturbation studied in this paper to be more easily generalizable to the latter kind of modes. A more complicated set of equations would have to be used to describe the former type of perturbation involving the "fluid" modes in the shell of matter outside the hole. While the calculation in this paper may not be easily generalizable to these more complicated situations involving gravitational waves coupled to matter, we note that a full perturbation treatment of the gravitational wave case will not be possible without a thorough understanding of the behavior of the Klein-Gordon wave equation with a perturbed potential, namely the system studied in this paper.

While the perturbed Klein-Gordon wave equation (1.3) is superficially similar to standard textbook problems, e.g., the usual Rayleigh-Schrödinger perturbation theory (RSPT), we note that the perturbation problem encountered here is fundamentally different: the outgoing wave condition renders the system physically nonconservative (energy escapes to infinity) and the associated operator $\left[-d^{2} / d x^{2}+V(x)\right]$ non-hermitian; hermiticity underpins the usual RSPT. 
The difficulty coming from the non-hermiticity can be seen in several guises if one tries naively to transcribe the usual formulas. Unlike the hermitian case, now the unperturbed eigenstates do not in general form a complete set for expansions [10,11], at least not in the case of black holes. The usual RSPT formula in terms of a sum over intermediate eigenstates is therefore inapplicable. Even the first-order shift, which does not involve a sum over intermediate states, cannot be given by the usual formula $\left\langle\phi_{0}\left|\mu V_{1}\right| \phi_{0}\right\rangle /\left\langle\phi_{0} \mid \phi_{0}\right\rangle$, in obvious notation - the usual inner product leads to $\left\langle\phi_{0} \mid \phi_{0}\right\rangle=\int_{-\infty}^{\infty} d x \phi_{0}^{*} \phi_{0}=\infty$ since a QNM wavefunction extends over all space (and indeed grows exponentially at infinity).

So far, the perturbation of black hole QNMs has attracted little attention, partly because a perturbative formalism for the QNMs of an open system, as opposed to the normal modes (NMs) of a conservative system, has not hitherto been available. In this paper we develop such a formalism, which then opens the way to extracting information about the astrophysical environment of the black holes from the observed signal, beyond the mass and the angular momentum of the hole.

This paper is a follow-up of [12], which outlined some of the results derived in this paper. In Sect. II of this paper, we develop a formulation for the perturbation of QNM systems. As a first step in this direction, we limit ourselves to the scalar wave case, in which the evolution is described by a single Klein-Gordon equation with a perturbed potential. The shifts in both the real and imaginary parts of the QNM frequencies $\omega$ are obtained in quadratures in terms of $\mu V_{1}(x)$, in principle to arbitrary order in $\mu$. Given the precision of the observational data that is possible in the near future (indeed at this point it is not clear how many QNM's one can extracted from the waveforms of black hole events, given the $\mathrm{S} / \mathrm{N}$ of even the advanced phase LIGO), the emphasis is on the first-order shift. The shifts when expressed in terms of a generalized inner product take a form similar to that in RSPT. The perturbative results for a Schwarzschild black hole are derived in Sect. III. We show that a function $H(x)$ can be defined which depends only on the original unperturbed system. We investigated and presented in detail the properties of this function $H$ for the black hole case. This function controls the phase and magnitude of the first-order shift of the spectrum for any given perturbation (not just for the model problem in this paper), hence providing insight to the properties of the black hole spectra in general. Sect. IV illustrates these results with a model problem where the perturbation is due to a spherical shell of matter located at a fixed radius, and we study scalar wave propagating in this background. The frequency shifts are obtained using the perturbation formula, and compared to numerical results. We show even in this simple case the spectra for shells located at different radii contain very interesting features. These features can be understood by the perturbation formula, demonstrating the power of the perturbation formula in providing understanding of the perturbed spectra.

\section{FORMULATION}

Waves defined by differential equations such as (1.3) or the optical analogs described by the wave equation [13] are open systems if the outgoing wave boundary condition is imposed; as such they are physically nonconservative and mathematically non-hermitian. The usual tools of mathematical physics based on the hermiticity of the defining operator 
would not, in general, be expected to apply. In attempting to develop generalizations of the familiar formalisms for conservative systems, it is important to recognize that these open systems fall into two broad classes. In the first, the potential $V(x)$ has discontinuities at $x=a_{1}, a_{2}$, and vanishes at infinity suitably fast ("no tail"); when these conditions are satisfied, the QNMs form a complete set on the interval $\left[a_{1}, a_{2}\right]$, and the usual formalism can be carried over with minimal changes [10,14. This is in itself somewhat surprising, in that the dynamics are controlled entirely by the resonances without the need to add any "background". However, when the discontinuity or "no-tail" conditions are not satisfied and this is the situation of interest in this paper - the QNMs are in general not complete, and the dynamics is not completely controlled by the QNMs. The QNMs are manifested in the complex frequency plane as poles of the Green's function; now, there is in addition a cut along the negative $\operatorname{Im} \omega$ axis [15]. The cut arises from scattering by the asymptotic part of the potential (in the sense that if $V(x)$ is truncated at any finite distance, the cut disappears). This is of course the case for the radial problem of a black hole, where $V(x)$ has no discontinuity and goes asymptotically as a centrifugal barrier plus $\sim \log x / x^{3}$; this tail generates the cut in the complex frequency plane, extending all the way to $\operatorname{Im} \omega \rightarrow 0$, and consequently leads to the long time power-law behavior $\sim t^{-\alpha}$ of the dynamics [15, 16]. The point to be stressed here is that since the QNMs are not complete, a perturbation formalism based on a sum over intermediate states will not be appropriate.

In this paper we need to rely on a different approach, which does not require a complete set of QNMs, by generalizing the logarithmic perturbation theory (LPT) [17 24], which focuses on the logarithmic derivative $f(x)=\phi^{\prime}(x) / \phi(x)$. Three features are relevant in the present context. First, the numerical determination of QNMs is known to be difficult, essentially because the exponentially growing solutions are numerically sensitive; this makes it important (indeed more important than would be the case for NMs) to develop analytic techniques, as well as semi-analytic techniques such as perturbation theory. Second, LPT does not require a complete set of unperturbed states for expansion, and is therefore well suited to situations where QNMs are not complete, or circumstances where only a few QNMs are known and others are not. (This is in practice often the case, since QNMs with large - Im $\omega$ are difficult to obtain, even numerically. The case of gravitational waves propagating away from a black hole is precisely of this type.) Thirdly, QNMs, being complex, are not in general plagued by nodes for real $x$, where $f(x)$ would be singular. In the case of NMs, the zeros of excited state wavefunctions require special treatment [21,23]; fortunately for QNMs, this is not a problem.

In terms of $f(x)$, (1.3) becomes the Riccati equation

-ricc

$$
f^{\prime}(x)+f^{2}(x)-\left[V_{0}(x)+\mu V_{1}(x)\right]+\omega^{2}=0 .
$$

The function $f(x)$ satisfies both the left boundary condition $(f(x) \rightarrow-i \omega$ as $x \rightarrow-\infty)$ and the right boundary condition $(f(x) \rightarrow+i \omega$ as $x \rightarrow+\infty)$, with $\omega$ in (2.1) being the eigenvalue $\omega$. However, for any $\omega$ (whether or not an eigenvalue), we can define two solutions $f_{ \pm}(\omega, x)$ by the boundary conditions $f_{ \pm}(\omega, x) \rightarrow \pm i \omega$ as $x \rightarrow \pm \infty$. At an eigenvalue $\omega$ $f_{+}(\omega, x)=f_{-}(\omega, x)=f(x)$. We expand the eigenvalue $\omega$ and the eigenfunction $f$ in powers of $\mu$ :

-omega 


$$
\omega=\omega_{0}+\mu \omega_{1}+\mu^{2} \omega_{2}+\cdots
$$

$-\mathrm{f}$

$$
f \equiv f_{0}+g=f_{0}+\mu g_{1}+\mu^{2} g_{2}+\cdots,
$$

where $f_{0}$, assumed known, satisfies the Riccati equation (2.1) with the potential $V_{0}$ and frequency $\omega_{0}$.

Putting (2.2) and (2.3) into the Riccati equation (2.1) and upon comparing powers of $\mu$, one finds, in a straightforward manner, that

-gn

$$
g_{n}^{\prime}+2 f_{0} g_{n}+2 \omega_{0} \omega_{n}=V_{n}
$$

for $n=1,2, \cdots$, in which $V_{1}$ is the perturbing potential in (1.3), and $V_{n}, n>1$, is the effective $n$th order potential, defined in terms of a combination of lower-order quantities:

$-\mathrm{vn}$

$$
V_{n}(x)=-\sum_{i=1}^{n-1}\left[g_{i}(x) g_{n-i}(x)+\omega_{i} \omega_{n-i}\right] .
$$

Using the integrating factor $\exp \left[2 \int d x f_{0}(x)\right]$, (2.4) can be solved; but since the resultant $g_{n}$, related to an eigenfunction, must satisfy two boundary conditions, this imposes a condition on the remaining free parameter $\omega_{n}$. The details of the derivation will be given elsewhere [24]. Ignoring convergence problems for the moment, and noting that the logarithmic derivatives at spatial infinity are unaffected by the perturbation, by definition of the boundary conditions, one obtains the following formal expressions, which constitute the core of LPT 25]:

-result

$$
\omega_{n}=\frac{\left\langle\phi_{0}\left|V_{n}\right| \phi_{0}\right\rangle}{2 \omega_{0}\left\langle\phi_{0} \mid \phi_{0}\right\rangle}
$$

Here we have introduced the suggestive notation

- matelm

$$
\left\langle\phi_{0}\left|V_{n}\right| \phi_{0}\right\rangle=\int_{-\infty}^{\infty} V_{n}(x) \phi_{0}^{2}(x) d x
$$

-norm

$$
\left\langle\phi_{0} \mid \phi_{0}\right\rangle=\int_{-\infty}^{\infty} \phi_{0}^{2}(x) d x
$$

These results express the $n$ th-order correction to the eigenvalue in quadrature in terms of lower-order quantities. The $n$ th-order correction to the logarithmic derivative is given by

-function

$$
g_{n}(x)=\left\{\int_{-\infty}^{x} d y\left[V_{n}(y)-2 \omega_{0} \omega_{n}\right] \phi_{0}^{2}(y)\right\} \phi_{0}^{-2}(x)
$$


When $g_{n}$ is substituted back in (2.5) to obtain $V_{n+1}$, the cycle of iteration is complete, and one can in principle obtain the corrections to any order. The strategy outlined here is basically the same as in the conventional LPT for hermitian systems.

The above expressions are formal and suggestive, but hide an essential problem: because the unperturbed wavefunctions go as $e^{ \pm i \omega_{0} x}$ as $x \rightarrow \pm \infty$, the integrands contain factors $\phi_{0}(x)^{2} \propto e^{2 \gamma_{0}|x|}$ as $|x| \rightarrow \infty\left(\gamma_{0}=-\operatorname{Im} \omega_{0}\right)$, rendering the integrals (2.7) and (2.8) divergent. It is therefore critical to handle the asymptotic regions carefully, which, as we shall see, may be regarded as a way of giving meaning to or regularizing these formally divergent expressions.

One can deal with the asymptotic regions in several slightly different ways [24], and we here give a simple version that relates most directly to the actual numerical implementation of the program. To do so, we return to (2.4), insert the integrating factor $\exp \left[2 \int d x f_{0}(x)\right]=$ $\phi_{0}^{2}(x)$, and integrate from $L_{-}$to $L_{+}$:

-int1

$$
\left.\phi_{0}^{2}(x) g_{n}(x)\right|_{L_{-}} ^{L_{+}}+2 \omega_{0} \omega_{n} \int_{L_{-}}^{L_{+}} d x \phi_{0}^{2}(x)=\int_{L_{-}}^{L_{+}} d x \phi_{0}^{2}(x) V(x) .
$$

(Had we taken $L_{ \pm}= \pm \infty$ instead of finite values, we would return to the formal result stated above.)

Now it has to be noticed that $g_{n}$ contains implicitly the unknown $n$th order frequency shift $\omega_{n}$. To see this, we note that $g\left(L_{ \pm}\right)$is to be matched to $f_{ \pm}\left(\omega, L_{ \pm}\right)-f_{0}\left(L_{ \pm}\right)$; consequently its $n$ th-order part is

- gnmatch

$$
\begin{aligned}
\mu^{n} g_{n} & =\left[f_{ \pm}\left(\omega, L_{ \pm}\right)-f_{0}\left(L_{ \pm}\right)\right]_{n}=\left[f_{ \pm}\left(\omega, L_{ \pm}\right)-f_{ \pm}\left(\omega_{0}, L_{ \pm}\right)\right]_{n} \\
& =\left[f_{ \pm}\left(\omega_{0}+\mu \omega_{1}+\cdots+\mu^{n-1} \omega_{n-1}, L_{ \pm}\right)-f_{ \pm}\left(\omega_{0}, L_{ \pm}\right)\right]_{n}+\mu^{n} \omega_{n} \frac{\partial}{\partial \omega} f_{ \pm}\left(\omega_{0}, L_{ \pm}\right) \\
& \equiv \mu^{n}\left[\Delta_{ \pm n}+\omega_{n} \frac{\partial}{\partial \omega} f_{ \pm}\left(\omega_{0}, L_{ \pm}\right)\right],
\end{aligned}
$$

where the subscript on the square brackets indicates taking the $n$ th-order part, and we have separated out the term that depends on $\omega_{n}$. The quantities $\Delta_{ \pm n}$ express the difference between two logarithmic derivatives at the asymptotic points. Thus, collecting the terms that do and do not contain $\omega_{n}$, we obtain from (2.10) again an expression like (2.6), except that the matrix element and norm are now given by

- matelm2

$$
\left\langle\phi_{0}\left|V_{n}\right| \phi_{0}\right\rangle=\int_{L_{-}}^{L_{+}} V_{n}(x) \phi_{0}^{2}(x) d x+\Delta_{-n} \phi_{0}^{2}\left(L_{-}\right)-\Delta_{+n} \phi_{0}^{2}\left(L_{+}\right)
$$

- norm2

$$
\left\langle\phi_{0} \mid \phi_{0}\right\rangle=\int_{L_{-}}^{L_{+}} \phi_{0}^{2}(x) d x+\frac{1}{2 \omega_{0}}\left[\phi_{0}^{2}\left(L_{+}\right) \frac{\partial}{\partial \omega} f_{+}\left(\omega_{0}, L_{+}\right)-\phi_{0}^{2}\left(L_{-}\right) \frac{\partial}{\partial \omega} f_{-}\left(\omega_{0}, L_{-}\right)\right] .
$$

Thus, provided we have a way of calculating the values of $f_{ \pm}\left(\omega, L_{ \pm}\right)$at the asymptotic positions $L_{ \pm}$, these alternate expressions $(2.12)$ and $(2.13)$ then provide practical ways of 
evaluating the matrix element and the norm, without infinite integrals or divergent expressions. In other words, the difficulties alluded to earlier have been eliminated. The choices of $L_{ \pm}$are arbitrary, and the shifts $\omega_{n}$ must be independent of these choices; in fact, it is easy to see that (2.12) and (2.13) must be separately independent of $L_{ \pm}$, simply because (2.13) relates only to the unperturbed state, whereas (2.12) depends on the arbitrary perturbation. This independence can be verified both analytically by differentiating the expressions with respect to $L_{ \pm}$and using the Riccati equation, or numerically by evaluating the expressions for different values of $L_{ \pm}$.

\section{GENERAL PROPERTIES}

The properties of the perturbed QNM's can be discussed at three levels of generality: (a) properties about open systems in general (in contrast to conservative systems); (b) properties for any perturbation of a Schwarzschild black hole; and (c) results for a specific perturbation. This Section deals with the first two, and a specific model of perturbation is presented in Sect. IV. Although the LPT is valid to all orders, for the present application we focus on the first-order shift.

\section{A. Open systems in general}

The result in (2.6) has been written in a way formally similar to the hermitian case. The factor $2 \omega_{0}$ occurs because the eigenvalue is $\omega^{2}$ rather than $\omega$. Since the numerator and the denominator in (2.6) are separately independent of $L_{ \pm}$, they can be given physical interpretations as a generalized matrix element and a generalized norm respectively.

The generalized norm has been introduced previously in a restricted form [26] applicable only to cases where the potential has "no tail"; in that case $\Delta_{ \pm}$can be obtained readily, since $f_{ \pm}\left(\omega, L_{ \pm}\right)= \pm i \omega$. In that restricted form, the perturbation theory for the QNMs of the wave equation has been developed and applied [27]. For systems such as black holes, the "no tail" condition must be removed, as sketched in Sect. II. In either case, the generalized norm has some unusual properties; (a) It involves $\phi_{0}^{2}$ rather than $\left|\phi_{0}\right|^{2}$, and is in general complex. (b) It involves surface terms at $x=L_{ \pm}$, though the value of the entire expression is independent of the choice of $L_{ \pm}$. Thus, it is not a norm in the strict sense, but rather a useful bilinear map. In cases where the system parameters can be tuned so that the leakage of the wavefunction approaches zero (e.g., $V_{0}(x)$ contains a tall barrier on both sides), then the generalized norm reduces to the usual (real and positive-definite) norm for a NM.

It is useful to define a function $H(x)$ for each QNM which depends only on the original unperturbed system:

- hdef

$$
\frac{\delta \omega}{\delta\left(\mu V_{1}(x)\right)} \equiv H(x)=\frac{\phi_{0}(x)^{2}}{2 \omega_{0}\left\langle\phi_{0} \mid \phi_{0}\right\rangle} .
$$

Both the magnitude and the phase of $H(x)$ are well defined and physically significant. The magnitude implies that we can now give a precise meaning to the normalization of a QNM, 
even though the wavefunction diverges at infinity. The phase of $H(x)$ determines the phase of the first-order shift $\omega_{1}$ for a real and positive localized perturbation $V_{1}(x)$. The phase is intriguing because it has no counterpart for a hermitian system - in that case, $H(x)$ must be real and non-negative. For an open system, $H(x)$ can be negative or indeed have any phase, so that a positive perturbation could lead to a decrease of the frequency.

The functions $H(x)$ are then convenient objects for discussing the effect of any perturbation on the QNMs of a given system. We next present some properties of $H(x)$ for the Schwarzschild black hole.

\section{B. Schwarzschild black holes}

First consider the normalization of these QNMs, made possible by the introduction of the generalized norm. Since (2.8) is independent of $L_{ \pm}$, it is particularly convenient if we take $L_{-}=L_{+}=0$ :

- defA

$$
\left\langle\phi_{0} \mid \phi_{0}\right\rangle=\frac{1}{2 \omega_{0}} \phi_{0}(0)^{2}\left\{\frac{\partial}{\partial \omega} f_{+}\left(\omega_{0}, 0\right)-\frac{\partial}{\partial \omega} f_{-}\left(\omega_{0}, 0\right)\right\} \equiv \phi_{0}^{2}(0) K .
$$

The logarithmic derivatives at $x=0$ can be obtained by integrating the differential equations from $x= \pm \infty$, and are readily calculated making use of Leaver's solution [28] (see Appendix B). The parameter $K$ is a convenient way of expressing the normalization, and Table $\square$ lists the values of $K /(2 M)$ for the lowest few QNMs (labeled by $j$ ) of the Regge-Wheeler potential for each angular momentum $l$. They increase as $j$ increases and show clear patterns, e.g., for $l=0, s=0 ; K$ is large for odd $j$, while for $l=2, s=2 ; K$ is large for even $j$. It is interesting to ask in what way the values of $K$ can characterize the Regge-Wheeler potentials, and to investigate their meaning beyond the perturbation calculation (noting that $K$ has nothing to do with the perturbation).

Next consider the functions $H(x)$. Fig. 2 shows $\operatorname{Re} H(x)$ and $\operatorname{Im} H(x)$ for $l=1$ scalar waves, versus the coordinate $x /(2 M)$. The diagrams refer to the lowest QNMs (labeled as $j=0,1, \cdots, 5)$ of that angular momentum. Since the wavefunctions grow exponentially as $|x| \rightarrow \infty$, we have multiplied $H(x)$ by $\exp \left[-2 \Gamma \sqrt{1+(x / 2 M)^{2}}\right]\left(\Gamma=-\operatorname{Im} 2 M \omega_{0}\right)$ for ease of plotting. These figures show an essentially plane wave behavior for large $|x|$, but the phases are nontrivial. We note that for a localized perturbation $\mu V_{1}(x)=\mu \delta\left(x-x_{1}\right)$, the firstorder shift in frequency $\omega_{1}$ is given by $H\left(x_{1}\right)$, and therefore can be read out directly from the figures. For example, Table II lists the magnitude and phases of $H(x)$ for $l=1, s=0$ and the lowest few $j$ 's at a fixed value $x=x_{1}\left(r=r_{1}\right)$, for $x_{1} / 2 M=0,5,10\left(r_{1} / 2 M=1.28\right.$, $3.93,8.05) ; r_{1} / 2 M=1.02$ and $1.08\left(x_{1} / 2 M=-2.89,-1.45\right)$. The patterns are different for these values of $x_{1}$ and not simple, demonstrating that a localized perturbation will push the QNMs along different directions in the complex frequency plane.

There is a more complicated structure in $H(x)$ for smaller $x$, best exhibited if the same data are plotted versus $r / 2 M$, as in Fig. 3. (The region $r / 2 M<2.0$ corresponds to $x / 2 M<$ 2.0.) The many oscillations for $x<0$ are compressed into a small region near $r / 2 M=1$ and are not visible in this plot. One interesting feature is that both $\operatorname{Re} H(x)$ and $\operatorname{Im} H(x)$ 
alternate in sign as $(-1)^{j}$ near the event horizon. The magnitude and phase of $H(x)$ at the sample positions $r=r_{1}$ where $r_{1} / 2 M=1.02\left(x_{1} / 2 M \approx-2.89\right)$ and $r_{1} / 2 M=1.08$ $\left(x_{1} / 2 M \approx-1.45\right)$ are also shown in Table $[1]$.

The results here described depend only on $H(x)$, i.e., on the properties of the unperturbed system. These examples indicate that localized perturbations can generate a rich pattern of frequency shifts (in contrast to shifts all of the same phase in the case of the NMs of a conservative system). In turn, this means that there are much better prospects of learning something about the perturbing potential from the observed shifts.

Of course, the richness of the pattern could be diluted if the perturbation is not localized in $x$, but has a spatial extent $\Delta x$ large compared to the typical wavelength $\lambda$ of oscillation of $H(x), \lambda \approx 2 \pi / \operatorname{Re} \omega_{0} \sim$ a few $M$ (see Fig. 2 for example). In the next Section we evaluate and discuss the perturbation that arises from the presence of a dust shell, which leads to a perturbation potential extending over a range of $x$.

\section{A MODEL PROBLEM}

\section{A. Description of the model}

We consider a Schwarzschild black hole surrounded by a static spherical shell of matter at a certain radius outside the hole. The equation of state of the matter making up the shell is chosen so that the shell remains static at the given radius. This example demonstrates that even such a simple model can lead to intriguing features in the QNM spectrum of the perturbed system.

There are two mass parameters in the black hole plus shell system: the total ADM mass of the system measured at infinity $M_{o}$, and the mass of the black hole as measured by its horizon surface area $M_{a}$ (i.e., the surface area of the event horizon is $16 \pi M_{a}^{2}$ ). In the limit of $M_{a} \rightarrow M_{o}$, we return to the unperturbed case of a bare black hole. Thus the parameter $\mu \equiv\left(M_{o}-M_{a}\right) / M_{a}$ is a measure of the perturbation. The perturbation further depends on the circumferential radius $r=r_{s}$ where the shell is placed. We shall study the shifts as a function of $\mu$ and $r_{s}$.

A static and spherically symmetric metric can be written as - metric

$$
d s^{2}=-A(r) d t^{2}+B(r) d r^{2}+r^{2}\left(d \theta^{2}+\sin ^{2} \theta^{2} d \varphi^{2}\right) .
$$

As usual, we define the mass function $m(r)$ such that

$$
g_{r r}=B(r)=\left[1-\frac{2 m(r)}{r}\right]^{-1} .
$$

The functions $m(r)$ and $A(r)$ satisfy the equations

$$
\frac{d m}{d r}=0 \quad, \quad r \neq r_{s}
$$




$$
\frac{1}{2 A} \frac{d A}{d r}=\frac{m}{r(r-2 m)}
$$

Hence we have

$$
\begin{gathered}
m(r)= \begin{cases}M_{o} & r>r_{s} \\
M_{a} & r<r_{s}\end{cases} \\
A(r)= \begin{cases}1-\frac{2 M_{o}}{r} & r \geq r_{s} \\
\alpha\left(1-\frac{2 M_{a}}{r}\right) & r<r_{s}\end{cases}
\end{gathered}
$$

where

$$
\alpha=\frac{1-2 M_{o} / r_{s}}{1-2 M_{a} / r_{s}} .
$$

The metric (4.1) becomes - metric2

$$
d s^{2}=\left\{\begin{array}{ll}
-\left(1-\frac{2 M_{o}}{r}\right) d t^{2}+\left(1-\frac{2 M_{o}}{r}\right)^{-1} d r^{2}+r^{2}\left(d \theta^{2}+\sin ^{2} \theta d \varphi^{2}\right) & r>r_{s} \\
-\alpha\left(1-\frac{2 M_{a}}{r}\right) d t^{2}+\left(1-\frac{2 M_{a}}{r}\right)^{-1} d r^{2}+r^{2}\left(d \theta^{2}+\sin ^{2} \theta d \varphi^{2}\right) & r<r_{s}
\end{array} .\right.
$$

The physical meanings of the parameters $r, M_{o}$ and $M_{a}$ are now clear from (4.8). We see explicitly that in the limit $M_{o} \rightarrow M_{a}$, the value of $\alpha$ reduces to unity, and (4.8) reduces to the familiar Schwarzschild metric.

Next we consider waves propagating on this perturbed Schwarzschild background. In this paper for simplicity we focus on the case of scalar wave $(s=0$ in (1.2)), described by

$$
\partial_{\mu} \partial^{\mu} \psi=\sqrt{-g} \partial_{\mu}\left(\sqrt{-g} g^{\mu \nu} \partial_{\nu} \psi\right)=0
$$

To obtain the Klein-Gordon equation suitable for the perturbation analysis, we separate $\psi$ into the radial and angular parts:

$$
\psi(\mathbf{r}, t)=\sum_{l=0}^{\infty} \sum_{m=-l}^{l} R_{l}(r, t) Y_{l m}(\theta, \varphi) .
$$

The radial function satisfies

$$
\frac{A}{B} R_{l}^{\prime \prime}+\left(\frac{2 A}{r B}+\frac{A^{\prime}}{2 B}-\frac{A B^{\prime}}{2 B^{2}}\right) R_{l}^{\prime}-\frac{A l(l+1)}{r^{2}} R_{l}=\partial_{t}^{2} R_{l},
$$

where ${ }^{\prime} \equiv d / d r$. Next introduce the tortoise coordinate

$$
x=\int^{r} \sqrt{\frac{B}{A}} d r
$$


to push the event horizon $\left(r=2 M_{a}\right)$ to $-\infty$. We leave the zero point of $x$ to be specified later. The equation for $R_{l}$ becomes

$$
\partial_{x}^{2} R_{l}+\frac{2}{r} \sqrt{\frac{A}{B}} \partial_{x} R_{l}-\frac{A l(l+1)}{r^{2}} R_{l}=\partial_{t}^{2} R_{l} .
$$

To cast the equation into the standard Klein-Gordon form, we introduce a function $\Phi(x, t)$ (hereafter the label $l$ will be suppressed) such that

$$
R(x, t)=h(x) \Phi(x, t)
$$

with

$$
\partial_{x} h+\frac{1}{r} \sqrt{\frac{A}{B}} h=0
$$

This function $\Phi(x, t)$ then satisfies the Klein-Gordon equation (1.1) with the effective potential

$$
V(r)=\frac{A l(l+1)}{r^{2}}+\frac{A^{\prime}}{2 B r}-\frac{A B^{\prime}}{2 r B^{2}}
$$

Substituting in the expressions for $A$ and $B$, we get

$$
V(r)= \begin{cases}\left(1-\frac{2 M_{o}}{r}\right)\left[\frac{l(l+1)}{r^{2}}+\frac{2 M_{o}}{r^{3}}\right] \equiv V_{S C}\left(M_{o}, r\right) & r>r_{s} \\ \kappa_{r} \delta\left(r-r_{s}\right) & r=r_{s} \\ \alpha\left(1-\frac{2 M_{a}}{r}\right)\left[\frac{l(l+1)}{r^{2}}+\frac{2 M_{a}}{r^{3}}\right]=\alpha V_{S C}\left(M_{a}, r\right) & r<r_{s}\end{cases}
$$

where $V_{S C}(M, r)$ is the standard unperturbed potential for a Schwarzschild black hole with mass $M$ (i.e., equation (1.2) with $s=0$ ). The strength of the $\delta$-function at the position of the shell $r_{s}$ is given by

$$
\kappa_{r}=\lim _{\epsilon \rightarrow 0^{+}} \int_{r_{s}-\epsilon}^{r_{s}+\epsilon} V(r) d r=-\left(1-\frac{2 M_{o}}{r_{s}}\right) \frac{\mu M_{a}}{r_{s}^{2}} .
$$

The remaining degree of freedom in setting up the perturbed problem is the zero-point of the tortoise coordinate $x$. We choose it so that the effective potential is the standard unperturbed form outside the shell, in terms of the ADM mass. The zero point is then determined by the requirement of continuity of $x$.

$$
x=\left\{\begin{array}{ll}
r+2 M_{o} \ln \left(r / 2 M_{o}-1\right) & r \geq r_{s} \\
\frac{1}{\sqrt{\alpha}}\left[r+2 M_{a} \ln \left(r / 2 M_{a}-1\right)\right]+x_{c} & r<r_{s}
\end{array},\right.
$$

where $x_{c}$ is the constant which makes $x$ continuous.

Thus we arrive at the perturbation problem (1.3) as discussed in the sections above with $x$ being the tortoise coordinate, 


$$
\begin{aligned}
V_{0}(x) & =V_{S C}\left(M_{o}, x\right) \\
\mu V_{1}(x) & = \begin{cases}0 & \text { for } x>x_{s} \\
\kappa \delta\left(x-x_{s}\right)+\alpha V_{S C}\left(M_{a}, r\right)-V_{S C}\left(M_{o}, r\right) & \text { otherwise }\end{cases}
\end{aligned}
$$

where

$$
x_{s}=r_{s}+2 M_{o} \ln \left(\frac{r_{s}}{2 M_{o}}-1\right)
$$

and

$$
\kappa=\lim _{\epsilon \rightarrow 0^{+}} \int_{x_{s}-\epsilon}^{x_{s}+\epsilon} V(x) d x=-\frac{2 \mu M_{a}}{r_{s}^{2}(1+1 / \sqrt{\alpha})} .
$$

This perturbation consists of two parts: a $\delta$-function at the position of the shell, plus a contribution inside the shell extending all the way to the horizon $\left(x \rightarrow-\infty\right.$ or $\left.r \rightarrow 2 M_{a}\right)$. There is no perturbation outside the shell.

We should make one further comment concerning the setup of the problem. As the equation of state of the matter shell is chosen to make the shell static, one need to ensure that the equation of state satisfies the energy conditions. It is straightforward to show that the dominant energy condition is the first to be violated if the shell is placed too close to the horizon, as shown in Appendix A. Thus, the discussion will be limited to the perturbations that do not violate the dominant energy condition.

\section{B. Numerical solution}

The full potential $V$ can be cast into a standard Regge-Wheeler potential for both $x<x_{s}$ and $x>x_{s}$, and hence exact QNMs can be obtained by numerically evaluating the Leaver's solutions of the Regge-Wheeler equation (see [28] and Appendix B]). The numerical solution will be used to examine the accuracy and validity of the perturbative result. Details of the numerical scheme are sketched as follows.

Firstly, as there is a $\delta$-function in the effective potential $V(x)$, the logarithmic derivatives at $x=x_{s}$ are related by

$$
\lim _{\epsilon \rightarrow 0^{+}}\left[\frac{\phi^{\prime}\left(\omega, x_{s}+\epsilon\right)}{\phi\left(\omega, x_{s}+\epsilon\right)}-\frac{\phi^{\prime}\left(\omega, x_{s}-\epsilon\right)}{\phi\left(\omega, x_{s}-\epsilon\right)}\right]=\kappa .
$$

Secondly, outside the shell, the effective potential is the Regge-Wheeler potential with mass parameter $M_{o}$ and the QNM wave function satisfies the outgoing wave boundary condition at the spatial infinity $x \rightarrow \infty$. Hence,

$$
\phi(\omega, x)=\phi_{+}\left(2 M_{o} \omega, x / 2 M_{o}\right),
$$

where $\phi_{+}(\tilde{\omega}, \tilde{x})$ is the outgoing wave solution of the scaled Klein-Gordon equation

$$
\left[\frac{d^{2}}{d \tilde{x}^{2}}+\tilde{\omega}^{2}-V(\tilde{r})\right] \phi(\tilde{\omega}, \tilde{x})=0
$$


with

$$
V(\tilde{r})=\left(1-\frac{1}{\tilde{r}}\right)\left[\frac{l(l+1)}{\tilde{r}^{2}}+\frac{1}{\tilde{r}^{3}}\right]
$$

and

$$
\tilde{x}=\tilde{r}+\ln (\tilde{r}-1) .
$$

Note that the quantities with tilde are dimensionless.

Thirdly, inside the shell, it is also easy to show that the wave function $\phi$ satisfies

$$
\left[\frac{d^{2}}{d \bar{x}^{2}}+\left(\frac{\omega}{\sqrt{\alpha}}\right)^{2}-V_{S C}\left(M_{a}, r\right)\right] \phi=0 \quad x<x_{s}
$$

and

$$
\bar{x}=r+2 M_{a} \ln \left(\frac{r}{2 M_{a}}-1\right)=\sqrt{\alpha}\left(x-x_{c}\right)
$$

Hence

$$
\phi(\omega, x)=\phi_{-}\left(2 M_{a} \omega / \sqrt{\alpha}, \bar{x} / 2 M_{a}\right) .
$$

Here $\phi_{-}(\tilde{\omega}, \tilde{x})$ is the ingoing wave solution of (4.26) at $\tilde{x} \rightarrow-\infty$.

Therefore, by connecting the two logarithmic derivatives at $x=x_{s}$ with (4.24), the QNM condition can be written as

$$
\left.\frac{\phi_{+}^{\prime}\left(2 M_{o} \omega, \tilde{x}\right)}{\phi_{+}\left(2 M_{o} \omega, \tilde{x}\right)}\right|_{\tilde{x}=x_{s} / 2 M_{o}}-\left.\frac{p \phi_{-}^{\prime}\left(2 M_{o} \omega / p, \tilde{x}\right)}{\phi_{-}\left(2 M_{o} \omega / p, \tilde{x}\right)}\right|_{\tilde{x}=\bar{x}_{s} / 2 M_{a}}=2 M_{o} \kappa
$$

where $p=\sqrt{\alpha} M_{o} / M_{a}$,

$$
\bar{x}_{s}=r_{s}+2 M_{a} \ln \left(\frac{r_{s}}{2 M_{a}}-1\right)=\sqrt{\alpha}\left(x_{s}-x_{c}\right),
$$

and the prime represents the derivative with respect to $\tilde{x}$. The functions $\phi_{+}$and $\phi_{-}$can be computed by the Leaver's solutions of the Regge-Wheeler equation (see 28 and Appendix B). Therefore, exact QNMs can be obtained by solving the nonlinear equation (4.32) by standard root-searching methods.

\section{Dependence on $\mu$ and convergence}

Given the perturbation function $\mu V_{1}$ in (4.21), the shifts $\omega_{1}$ and $\omega_{2}$ can be evaluated by (2.6). The detailed treatments will be given in Appendix 9 .

Fig. 4 shows the 0th, 1st and 2nd order perturbation results for $l=1$ scalar waves together with the exact numerical results for a black hole plus shell system. The parameters are $\mu=0.02, r_{s}=2.52 M_{a}\left(x_{s} \approx 0\right)$. This figure shows that the perturbation formalism does give the correct shifts for small $\mu$.

To see the convergence more clearly, we plot in Fig. 5 the magnitude of the error in the frequencies in the 0 th, 1 st and 2 nd order results versus $\mu$. The plot shows the case of $l=1$, $s=0, j=1$ (first excited state), with the shell located at $r_{s}=2.52 M_{a}$. The error of the $n$ th-order result goes as $\mu^{n+1}$, as it should. 


\section{Dependence on shell position}

We next study the dependence on the parameters of the shell. Fig. 6 shows the trajectories of the lowest damping QNMs $(j=0,1, \cdots 6)$ as the position of the shell moves away from the event horizon. The plot shows the case of $l=1$ scalar waves with $\mu=0.01$ based on numerical calculation. The regions near the $j=0$ and $j=1$ modes are shown in greater detail in the insets. The calculation is terminated when the QNM is near the imaginary $\omega$ axis, since it is difficult to perform the numerical calculation with sufficient accuracy in that region. It is seen that there are interesting changes in signs with increasing $j$. Some QNMs move toward the imaginary $\omega$ axis, and the higher damping QNMs either move toward the imaginary $\omega$ axis or move upward toward the origin. It is also seen that when the shell is placed far away from the event horizon, the QNMs move away from their unperturbed positions, with the higher damping modes moving with higher speed. This behavior can be understood from the perturbation formula (2.6). When $x_{s} / 2 M_{a} \gg 1$, we have, according to (4.21) and (2.6), 一 asyom

$$
\omega_{1} \sim e^{2 i \omega_{0} x_{s}} / x_{s}^{2} \quad \text { for } \quad x_{s} / 2 M_{a} \gg 1 .
$$

Hence QNMs move more rapidly away from the unperturbed positions when the shell is far away from the event horizon $\left(x_{s} / 2 M_{a} \gg 1\right)$ and the higher damping modes $\left(-\operatorname{Im} 2 M_{a} \omega_{0} \gg 1\right)$ move with higher speed.

Fig. 7 shows the first-order perturbation result for the $j=0$ mode for different values of $x_{s}$. At large $x_{s}$, the trajectory shows a spiral structure, which can be explained from the first-order perturbation formula. According to (4.34),

$$
\frac{d \omega}{d x_{s}} \approx \frac{d \omega_{1}}{d x_{s}} \sim \frac{e^{2 i \omega_{0} x_{s}}}{x_{s}^{2}} \quad \text { for } x_{s} \gg 1 .
$$

The exponential factor $e^{2 i \omega_{0} x_{s}}$ leads to the spiral structure. For larger $j$ modes, there is no such spiral structure (Fig. 6) because the higher-order corrections become large before the spiral appears, as shown in Fig. 8.

\section{CONCLUSION}

We have applied the LPT for the QNMs of open systems to the study of gravitational waves propagating away from black holes. QNM gravitational wave signals from black holes will be detected soon, and many black holes are expected to be perturbed by their astrophysical environment, e.g., by an accretion disk. The study in this paper on scalar waves represents the first step in the study of the QNM spectra of waves propagating in a dirty black hole background, perturbed by the astrophysical environment. Its further development can be of interest to gravitational wave astronomy, among other applications.

Although the QNMs of any system can in principle be obtained through brute force numerical integration, it is nevertheless highly desirable to have a convenient set of perturbation formulae that one can understand the system with. We note that the Rayleigh-Schrödinger 
perturbation theory for NMs is tremendously valuable, even though the NM systems are more easily handled numerically than QNM systems.

We have shown in a simple example that a perturbed black hole spectrum can have interesting features, and demonstrated how these features can be understood with the perturbation formula. In summary, we raise the importance of studying QNMs of dirty black holes, and show how it can be done in a perturbative formulation.

\section{ACKNOWLEDGMENTS}

This work is supported in part by the Hong Kong Research Grants Council grant 452/95P and the US NSF grant PHY 96-00507 and NASA grant NCCS5-153. WMS also thanks the

Institute of Mathematical Science of The Chinese University of Hong Kong for its support.

\section{APPENDIX A: DOMINANT ENERGY CONDITION}

The shell cannot be placed too near to the black hole; otherwise, the dominant energy condition

$$
\left.\left|S_{\hat{t}}^{\hat{t}}\right|>\left|S_{\hat{q}}^{\hat{q}}\right| \quad \text { (no sum on } q\right)
$$

will be violated, where $\hat{q}$ denotes any unit specelike vector, and

$$
S_{\hat{\mu}}^{\hat{\nu}}=\lim _{\epsilon \rightarrow 0^{+}} \int_{r_{s}-\epsilon}^{r_{s}+\epsilon} T_{\hat{\mu}}^{\hat{\nu}} d \hat{r} .
$$

It is straightforward to compute all the non-trivial components of the Einstein tensor for the metric (4.1):

$$
\begin{gathered}
G_{r}{ }^{r}=\frac{A^{\prime}}{r A B}+\frac{1}{r^{2} B}-\frac{1}{r^{2}} \\
G_{\theta}{ }^{\theta}=G_{\varphi}{ }^{\varphi}=\frac{A^{\prime}}{2 r A B}-\frac{A^{\prime 2}}{4 A^{2} B}-\frac{B^{\prime}}{2 r B^{2}}-\frac{A^{\prime} B^{\prime}}{4 A B^{2}}+\frac{A^{\prime \prime}}{2 A B} \\
G_{t}{ }^{t}=\frac{1}{r^{2} B}-\frac{1}{r^{2}}-\frac{B^{\prime}}{r B^{2}},
\end{gathered}
$$

where ${ }^{\prime} \equiv d / d r$. The component $G_{\mu}{ }^{\nu}$ is non-zero only if it contains terms involving the second derivative of $A$ or the first derivative of $B$. Hence

$$
\begin{aligned}
S_{\hat{t}}^{\hat{t}}=S_{t}^{t} & =\frac{1}{8 \pi} \lim _{\epsilon \rightarrow 0^{+}} \int_{r_{s}-\epsilon}^{r_{s}+\epsilon} \frac{-B^{\prime}}{r B^{3 / 2}} d r \\
& =-\frac{M_{o}-M_{a}}{2 \pi r_{s}^{2}}\left(\sqrt{1-\frac{2 M_{o}}{r_{s}}}+\sqrt{1-\frac{2 M_{a}}{r_{s}}}\right)^{-1}
\end{aligned}
$$

using the Einstein equations $G_{\mu}{ }^{\nu}=8 \pi T_{\mu}{ }^{\nu}$. Similarly, we have 


$$
S_{\hat{\theta}}^{\hat{\theta}}=S_{\hat{\phi}}^{\hat{\phi}}=\frac{1}{8 \pi r_{s}}\left(\frac{1-M_{o} / r_{s}}{\sqrt{1-2 M_{o} / r_{s}}}-\frac{1-M_{a} / r_{s}}{\sqrt{1-2 M_{a} / r_{s}}}\right) .
$$

(A1) together with (A7) and (A8) imply a lower bound on $r_{s}$ for a given $\mu$. For $\mu=0.01$, it is found that the minimum $r_{s}$ is $2.26 M_{a}$.

\section{APPENDIX B: LEAVER'S SOLUTIONS}

In the analytic study of the Regge-Wheeler equation, it is convenient to rewrite the Regge-Wheeler equation in terms of the coordinate $r$ :

$$
r(r-1) \phi_{, r r}+\phi_{, r}-\left[\frac{\rho^{2} r^{3}}{r-1}+l(l+1)+\frac{\xi}{r}\right] \phi=0,
$$

where $\rho=-i \omega, \xi=1-s^{2}$. For simplicity, we have set $2 M=1$. The equation (B1) can be transformed to the generalized spheroidal wave equation [28]. For an arbitrary frequency $\omega$, we define two solutions $\phi_{ \pm}(\omega, r)$ which satisfy the boundary conditions

$$
\phi_{ \pm} \propto e^{ \pm i \omega x} \quad \text { for } x \rightarrow \pm \infty
$$

The analytic solutions of these two functions are given by [28]

$$
\begin{aligned}
& \phi_{-}(\omega, r)=(r-1)^{\rho} r^{-2 \rho} e^{-\rho r} \sum_{n=0}^{\infty} a_{n}\left(\frac{r-1}{r}\right)^{n} \\
& \phi_{+}(\omega, r)=r^{1+s}(r-1)^{\rho} e^{-\rho r} \sum_{n=0}^{\infty} a_{n}(2 \rho+1)_{n} U(s+1+2 \rho+n, 2 s+1,2 \rho r),
\end{aligned}
$$

where

$$
(2 \rho+1)_{n} \equiv \frac{\Gamma(2 \rho+1+n)}{\Gamma(2 \rho+1)}
$$

is the Pochhammer's symbol, $U$ is the irregular confluent hypergeometric function [30], and $a_{n}$ is determined by the following three-term recursion relation:

$$
\alpha_{n} a_{n+1}+\beta_{n} a_{n}+\gamma_{n} a_{n-1}=0 \quad n=1,2, \cdots,
$$

with

$$
a_{n}=0 \quad \text { for } n<0 .
$$

The value of $a_{0}$ is arbitrary and is related to the normalization of $\phi_{ \pm}$. The quantities $\alpha_{n}$, $\beta_{n}$ and $\gamma_{n}$ are defined to be

$$
\begin{aligned}
\alpha_{n} & =(n+1)(n+2 \rho+1) \\
\beta_{n} & =-\left[2 n^{2}+(8 \rho+2) n+8 \rho^{2}+4 \rho+l(l+1)+\xi\right] \\
\gamma_{n} & =n^{2}+4 \rho n+4 \rho^{2}+\xi-1 .
\end{aligned}
$$


In most cases, we only need the logarithmic derivatives

$$
f_{ \pm}(\omega, x)=\frac{1}{\phi_{ \pm}} \frac{d \phi_{ \pm}}{d x}
$$

While $f_{-}(\omega, x)$ can be easily obtained from $(\mathbb{B} 3)$, the calculation of $f_{+}(\omega, x)$ is less straightforward as it involves a sum of irregular confluent hypergeometric functions, which are notoriously different to evaluate [28]. In the following, we develop a numerical algorithm to calculate $f_{+}(\omega, x)$.

We define two sequences

$$
\begin{aligned}
& T_{n}^{(-)}=(2 \rho+1)_{n} U(s+1+2 \rho+n, 2 s+1,2 \rho r) \\
& T_{n}^{(+)}=\frac{\Gamma(2 \rho+1+n)}{\Gamma(2 \rho+n-s+1)} M(s+1+2 \rho+n, 2 s+1,2 \rho r)
\end{aligned}
$$

where $M$ is the regular confluent hypergeometric function. Hence we have

$$
\phi_{+}(\omega, r)=r^{1+s}(r-1)^{\rho} e^{-\rho r} h(\omega, r)
$$

where

$$
h(\omega, r)=\sum_{n=0}^{\infty} a_{n} T_{n}^{(-)}
$$

$f_{+}(\omega, x)$ can then be expressed in terms of $h$ as follows:

$$
f_{+}(\omega, x)=\left(1-\frac{1}{r}\right) \frac{1}{\phi_{+}} \frac{d \phi_{+}}{d r}=\left(1-\frac{1}{r}\right)\left[\frac{1+s}{r}+\frac{\rho}{r-1}-\rho+\frac{1}{h} \frac{d h}{d r}\right] .
$$

It can be shown, from the recursion formulae of the confluent hypergeometric functions [30,31, that

$$
\begin{aligned}
& (2 \rho+n)(2 \rho+n+1) T_{n-1}^{( \pm)}-(2 \rho+n+1)(1+4 \rho+2 n+2 \rho r) T_{n}^{( \pm)} \\
& +(s+1+2 \rho+n)(2 \rho+n-s+1) T_{n+1}^{( \pm)}=0
\end{aligned}
$$

and

$$
\frac{d T_{n}^{(-)}}{d r}=\frac{s+1+2 \rho+n}{r}\left[\frac{2 \rho+n+1-s}{2 \rho+n+1} T_{n+1}^{(-)}-T_{n}^{(-)}\right] .
$$

For large $n, T_{n}^{( \pm)}$approaches the asymptotic expressions [31]

$$
\begin{aligned}
& T_{n}^{(-)} \approx \frac{\sqrt{\pi} \Gamma(2 \rho+1+n)}{\Gamma(2 \rho+1) \Gamma(2 \rho+1+n+s)}\left(\frac{n}{2 \rho r}\right)^{s}(2 n \rho r)^{-1 / 4} e^{\rho r} \exp \left[-2(2 n \rho r)^{1 / 2}\right] \\
& T_{n}^{(+)} \approx \frac{\Gamma(2 s+1) \Gamma(2 \rho+1+n)}{2 \sqrt{\pi} \Gamma(2 \rho+1+s+n)}\left(\frac{n}{2 \rho r}\right)^{s}(2 n \rho r)^{-1 / 4} e^{\rho r} \exp \left[+2(2 n \rho r)^{1 / 2}\right] .
\end{aligned}
$$


The general solution of the difference equation $(\mathrm{B} 15)$ is a linear combination of $T_{n}^{( \pm)}$. It follows from $(\mathrm{B} 17)$ and $(\mathrm{B} 18)$ that $T_{n}^{(+)}$increases with $n$, while $T_{n}^{(-)}$decreases with $n$. Hence any linear combination of $T_{n}^{( \pm)}$will eventually be dominated by the term containing $T_{n}^{(+)}$as $n \rightarrow \infty$. The computation of $T_{n+1}^{(-)}$from $T_{n}^{(-)}$and $T_{n-1}^{(-)}$is therefore unstable. The recursion relation $(\mathrm{B} 15)$ must be used in the reverse direction. In practice, we choose an arbitrary value of $T_{N}^{(-)}(N \gg 1)$ and set

$$
T_{N-1}^{(-)}=\frac{T_{N-1}^{(-)}}{T_{N}^{(-)}} \times T_{N}^{(-)} \approx T_{N}^{(-)} \frac{2 \rho+N+s}{2 \rho+N}\left(\frac{N-1}{N}\right)^{s-1 / 4} \exp \left[\frac{2(2 \rho r)^{1 / 2}}{\sqrt{N}+\sqrt{N-1}}\right] .
$$

The values of $T_{n}^{(-)}$for $n<N-1$ are then calculated by the recursion formula (B15). $T_{n}^{(-)}$ determined in this way will differ from its original definition in (B10) by a multiplicative constant. This does not concern us since we are only interested in the logarithmic derivative of $\phi_{+}$, which is independent of the constant factor and is readily evaluated by (B14).

Leaver's solutions can be applied to compute the logarithmic derivatives $f_{0}\left(L_{ \pm}\right)$in (2.13) and to evaluate the QNMs of the shell model of dirty black hole described in Sect. IV.

\section{APPENDIX C: EVALUATION OF THE PERTURBATION FORMULAE}

For simplicity we focus on the first order perturbation calculation, which is given by

$$
\omega_{1}=\frac{\left\langle\phi_{0}\left|V_{1}\right| \phi_{0}\right\rangle}{2 \omega_{0}\left\langle\phi_{0} \mid \phi_{0}\right\rangle}
$$

We have discussed the evaluation of the generalized norm $\left\langle\phi_{0} \mid \phi_{0}\right\rangle$ in Sect. IIIB. It suffices to evaluate the generalized matrix element $\left\langle\phi_{0}\left|V_{1}\right| \phi_{0}\right\rangle$. For $V_{1}$ given by (4.21), the generalized matrix element is

$$
\left\langle\phi_{0}\left|V_{1}\right| \phi_{0}\right\rangle=\int_{L_{-}}^{x_{s}} V_{1}(x) \phi_{0}^{2}(x) d x+\kappa \phi_{0}\left(x_{s}\right)^{2}+\left.\phi_{0}^{2}\left(L_{-}\right) \frac{\partial}{\partial \mu} f_{-}\left(\omega_{0}, L_{-}\right)\right|_{\mu=0},
$$

where $L_{-}$is any real number smaller than $x_{s}$. It has been argued earlier that this expression is independent of the choice of $L_{-}$, and for any finite $L_{-}$the expression is finite. Thus the entire perturbation scheme requires no regularization.

However, the last term in (C2) involves the change of $f_{-}$due to the presence of $V_{1}$. This calculation can be bypassed by the following trick. First, since the entire expression is independent of $L_{-}$, we push $L_{-}$to $-\infty$ and define a function

$$
Z(\omega)=\lim _{L_{-} \rightarrow-\infty}\left[\int_{L_{-}}^{x_{s}} \phi_{0-}^{2}\left(\omega, r_{*}\right) V_{1}\left(r_{*}\right) d r_{*}+\left.\phi_{0-}^{2}\left(\omega, L_{-}\right) \frac{\partial}{\partial \mu} f_{-}\left(\omega, L_{-}\right)\right|_{\mu=0}\right]+\kappa \phi_{0-}^{2}\left(\omega, x_{s}\right),
$$

where $\phi_{0-}\left(\omega, r_{*}\right)$ is a function satisfying the unperturbed Regge-Wheeler equation with the ingoing wave boundary condition on the event horizon. This expression as a whole is finite for every $\omega$, but for $\operatorname{Im}(\omega)>0$, each term is separately finite; in fact the surface term 
vanishes as $L_{-} \rightarrow-\infty$ because the factor $\phi_{0-}^{2}\left(\omega, L_{-}\right) \propto e^{-2 i \omega L_{-}}$now decays exponentially. Evaluating in this domain, we have

$$
Z(\omega)=\int_{-\infty}^{x_{s}} \phi_{0-}^{2}\left(\omega, r_{*}\right) V_{1}\left(r_{*}\right) d r_{*}+\kappa \phi_{0-}^{2}\left(\omega, x_{s}\right) \quad \text { for } \operatorname{Im}(\omega)>0 .
$$

The prescription is therefore to evaluate in this domain and analytically continue to $\omega_{0}$ :

$$
\left\langle\phi_{0}\left|V_{1}\right| \phi_{0}\right\rangle=Z\left(\omega_{0}\right)=\int_{-\infty}^{x_{s}} \phi_{0}^{2}\left(r_{*}\right) V_{1}\left(r_{*}\right) d r_{*}+\kappa \phi_{0}^{2}\left(x_{s}\right)
$$

We note that this merely provides an alternate evaluation of an expression that was manifestly finite to start with.

The integral can be easily carried out analytically by noticing that the potential $V_{S C}(M, x)$ in (4.17) can be expressed as a sum of exponentials at negative $x$; the calculation is sketched below and the details of the straightforward arithmetic can be found in Ref. [32]:

$$
\begin{aligned}
V_{S C}(M, x) & =\frac{1}{4 M^{2}} V_{S C}\left(\frac{1}{2}, \frac{x}{2 M}\right) \\
V_{S C}(1 / 2, x) & =\sum_{k=1}^{\infty} c_{k} e^{k \tilde{x}} \quad \text { for } \tilde{x}<0,
\end{aligned}
$$

where

$$
\begin{aligned}
c_{k} & =l(l+1) \gamma_{3, k}+\left(1-s^{2}\right) \gamma_{4, k} \\
\gamma_{n, m} & =e^{-m} \sum_{p=0}^{m} \mu(p, n, m) .
\end{aligned}
$$

Here $\mu(0, n, 1)=1-n, \mu(1, n, 1)=n, \mu(p, n, m)=0$ for $p<0$ and $p>m$, and

$\mu(p, n, m)=-\frac{(n+p+m-2) \mu(p-1, n, m-1)+(m-1) \mu(p, n, m-1)}{m} \quad$ for $m \geq 2$.

It can be shown that the unperturbed wave function $\phi_{0}$ can be expressed as 29

$$
\phi_{0}(x)=\sum_{k=0}^{\infty} d_{k}\left(\omega_{0}\right) e^{k x /\left(2 M_{0}\right)}
$$

where

$$
d_{k}\left(\omega_{0}\right)=\frac{1}{k\left(k-2 i \omega_{0}\right)} \sum_{m=0}^{k-1} d_{k}\left(\omega_{0}\right) c_{k-m},
$$

and $d_{0}$ is a constant related to the normalization of the wavefunction.

It is obvious that $V_{1}$ given by (4.21) can also be expressed as a sum of exponentials plus a $\delta$-function. Hence the integration in (C2) can be performed analytically, giving the generalized matrix element and the first-order shift $\omega_{1}$. 


\section{REFERENCES}

[1] See, e.g., A. A. Abramovici et. al., Science 256, 325 (1992).

[2] C. V. Vishveshwara, Nature (London) 227, 937 (1970); S. L. Detweiler and E. Szedenits, Astrophys. J. 231, 211 (1979); L. Smarr, in Sources of Gravitational Radiation, edited by L. Smarr (Cambridge Univ. Press, Cambridge, England, 1979); R. F. Stark and T. Piran, Phys. Rev. Lett. 55, 891 (1985).

[3] See e.g., P. Anninos et. al., Phys. Rev. Lett. 71, 2851 (1993) and references therein.

[4] See e.g., S. Chandrasekhar, The Mathematical Theory of Black Holes (Oxford U. Press, 1983).

[5] See e.g., S. W. Hawking and G. F. R. Ellis, The Large Scale Structure of Spacetime (Cambridge U. Press, 1973).

[6] T. Regge and J. A. Wheeler, Phys. Rev. 108, 1063 (1957).

[7] S. Detweiler, Astrophys. J. 239, 292 (1980); V. Ferrari and B. Mashhoon, Phys. Rev. Lett. 52, 1361 (1984); B. F. Schutz and C. M. Will, Astrophys. J. 291, L33 (1985); H. Liu and B. Mashhoon, Class. Quantum Grav. 13, 233 (1996).

[8] E. W. Leaver, Proc. R. Soc. London A 402, 285 (1985); J. W. Guinn, C. M. Will, Y. Kojima and B. F. Schutz, Class. Quantum Grav. 7, L47 (1990); E. Leaver, Class. Quantum Grav. 9, 1643 (1992); F. Andersson and S. Linnaeus, Phys. Rev. D 46, 4179 (1992); H.-P. Nollert, Phys. Rev. D 47, 5253 (1993).

[9] H. Onoazawa, Phys. Rev. D 55, 3593 (1997).

[10] E. S. C. Ching, P. T. Leung, W. M. Suen, and K. Young, Phys. Rev. Lett. 74, 4588 (1995).

[11] E. S. C. Ching, P. T. Leung, W. M. Suen, and K. Young, Phys. Rev. D 54, 3778 (1996).

[12] P. T. Leung, Y. T. Liu, W.-M. Suen, C. Y. Tam, and K. Young, Phys. Rev. Lett. 78, 2894 (1997).

[13] P. T. Leung, S. Y. Liu and K. Young, Phys. Rev. A 49, 3057 (1994); P. T. Leung, S. Y. Liu, S. S. Tong and K. Young, Phys. Rev. A 49, 3068 (1994); P. T. Leung, S. Y. Liu and K. Young, Phys. Rev. A 49, 3982 (1994).

[14] P. T. Leung, S. S. Tong, and K. Young, J. Phys. A 30, 2139 (1997). P. T. Leung, S. S. Tong, and K. Young, J. Phys. A 30, 2153 (1997).

[15] E. W. Leaver, Phys. Rev. D 34, 384 (1986); E. S. C. Ching, P.T. Leung, W.M. Suen, and K. Young, Phys. Rev. Lett. 74, 2414 (1995); E. S. C. Ching, P.T. Leung, W.M. Suen, and K. Young, Phys. Rev. D 52, 2118 (1995).

[16] R. H. Price, Phys. Rev. D 5, 2419 (1972); R. H. Price, Phys. Rev. D 5, 2439 (1972).

[17] G. Wentzel, Z. Phys. 38, 518 (1926).

[18] R. J. Price, Proc. Phys. Soc. 67, 383 (1954).

[19] V. S. Polikanov, Zh. Eksp. Teor. Fiz. 52, 1326 (1967) [Soc. Phys. JETP 25, 882 (1967)]; Theor. Math. Phys. (USSR) 24, 230 (1975).

[20] Y. Aharonov and C. K. Au, Phys. Rev. Lett. 42, 1582 (1979).

[21] C. K. Au, Phys. Rev. A 29, 1034 (1984).

[22] Y. Aharonov and C.K. Au, Phys. Rev. A 20, 2245 (1979); C. K. Au, Phys Rev A 29, 1034 (1984).

[23] C. K. Au et. al., J. Phys. A 24, 3837 (1991).

[24] P. T. Leung et. al., J. Phys. A 31, 3271 (1998). 
[25] The "extraneous" factor of $2 \omega_{0}$ (compared to the usual result for the Schrödinger equation) occurs because the eigenvalue is $\omega^{2}$, and $\Delta\left(\omega^{2}\right)=2 \omega \Delta \omega$,

[26] For the particular case of potentials with both $V_{0}$ and $V_{1}$ having finite support, the generalized norm has been introduced previously both for the wave equation [13], the Schrödinger equation [33] and the Klein-Gordon equation [11], and its property discussed. An equivalent form first given by Zeldovich [34] did not have the surface terms, but instead required a process of regularization; it is less convenient when actual evaluation (especially numerical evaluation) is called for. The present result, in the more general form (2.8), is however applicable to potentials that extend to infinity, including, for example, the important Regge-Wheeler potential (1.2) to be discussed below.

[27] P. T. Leung, S. Y. Liu, S. S. Tong and K. Young, Phys. Rev. A 49, 3068 (1994); P. T. Leung, S. Y. Liu and K. Young, Phys. Rev. A 49, 3982 (1994).

[28] E. W. Leaver, J. Math. Phys. 27, 1238 (1986).

[29] P. T. Leung et. al., Phys. Lett. A, in press.

[30] M. Abramowitz and I. A. Stegun, Handbook of Mathematical Functions (Dover 1970).

[31] N. M. Temme, Numer. Math. 41, 63 (1983).

[32] Y. T. Liu, A Study of Quasinormal Modes of Black Holes, M. Phil. thesis, The Chinese University of Hong Kong (unpublished).

[33] P. T. Leung and K. Young, Phys. Rev. A 44, 3152 (1991).

[34] Ya. B. Zeldovich, Zh. Eksp. Teor. Fiz. 39, 776 (1960) [Sov. Phys. JETP 12, 542 (1961)]. 


\section{FIGURES}

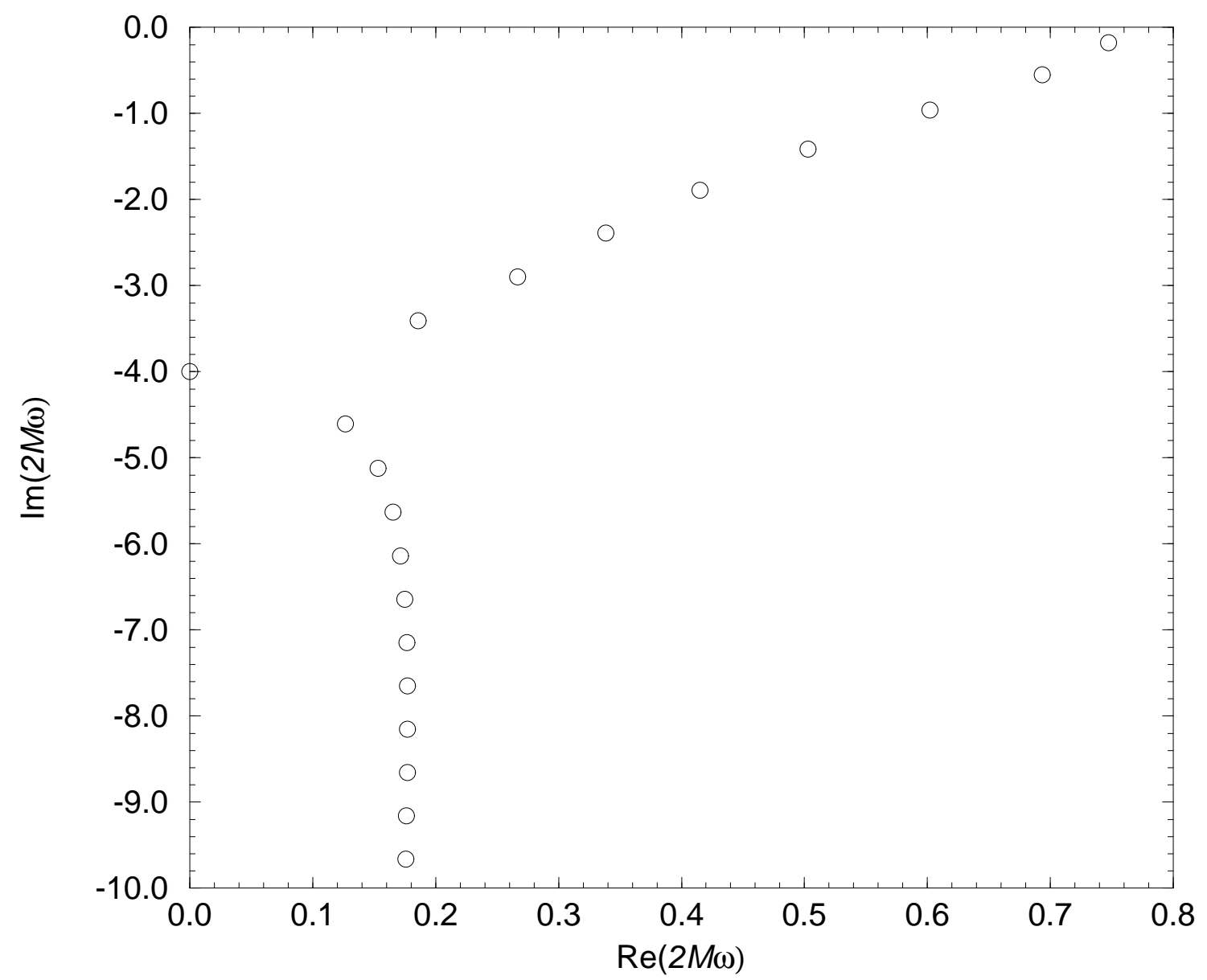

FIG. 1. The distribution of QNMs of a Schwarzschild black hole for $l=s=2$. 

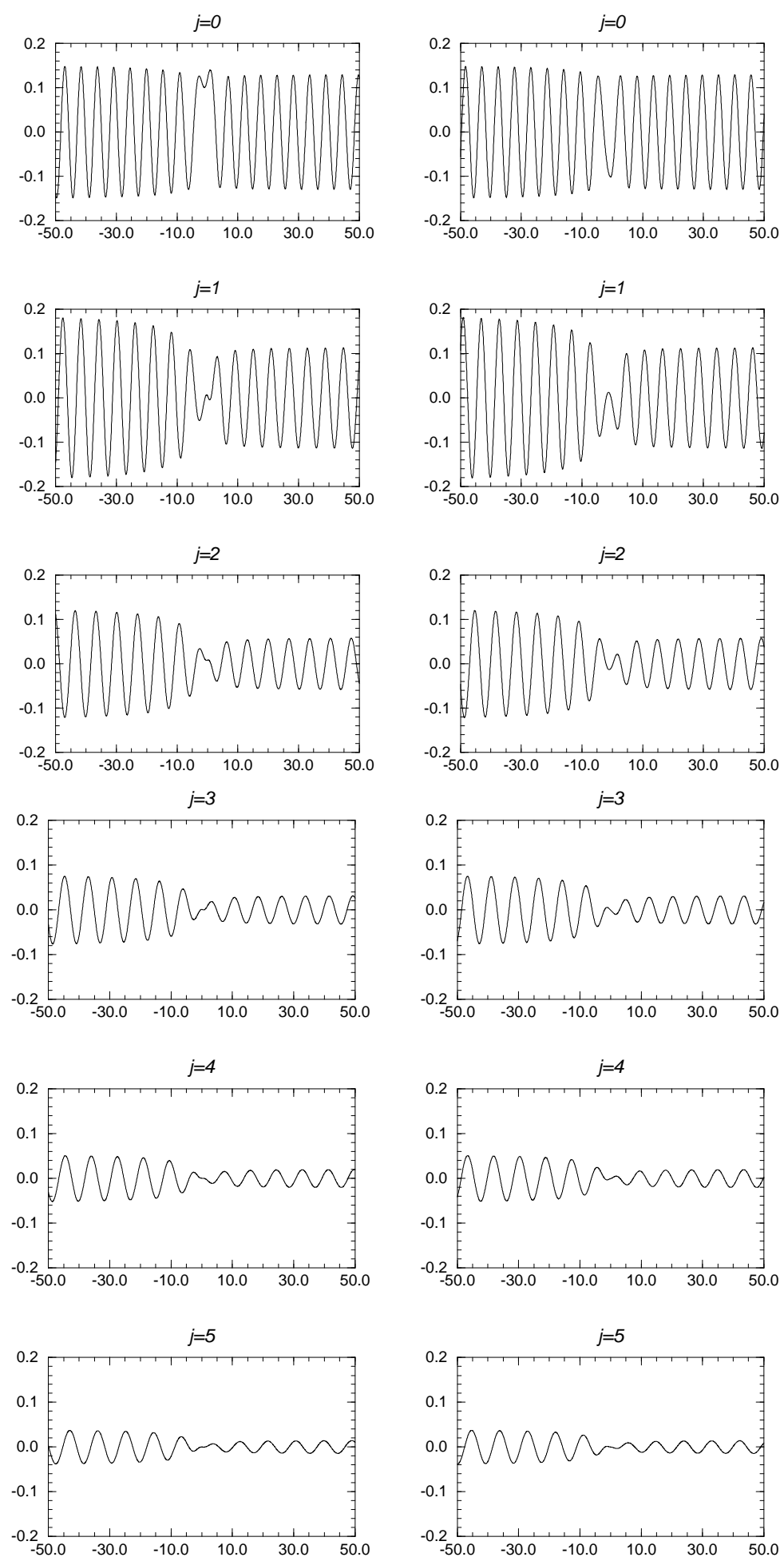

FIG. 2. Graph of $\operatorname{Re} H(x) e^{-2 \Gamma \sqrt{1+(x / 2 M)^{2}}}$ (left) and $\operatorname{Im~} H(x) e^{-2 \Gamma \sqrt{1+(x / 2 M)^{2}}}$ (right) vs $x / 2 M$ for $l=1, s=0$, and $j=0,1, \cdots 6$. 

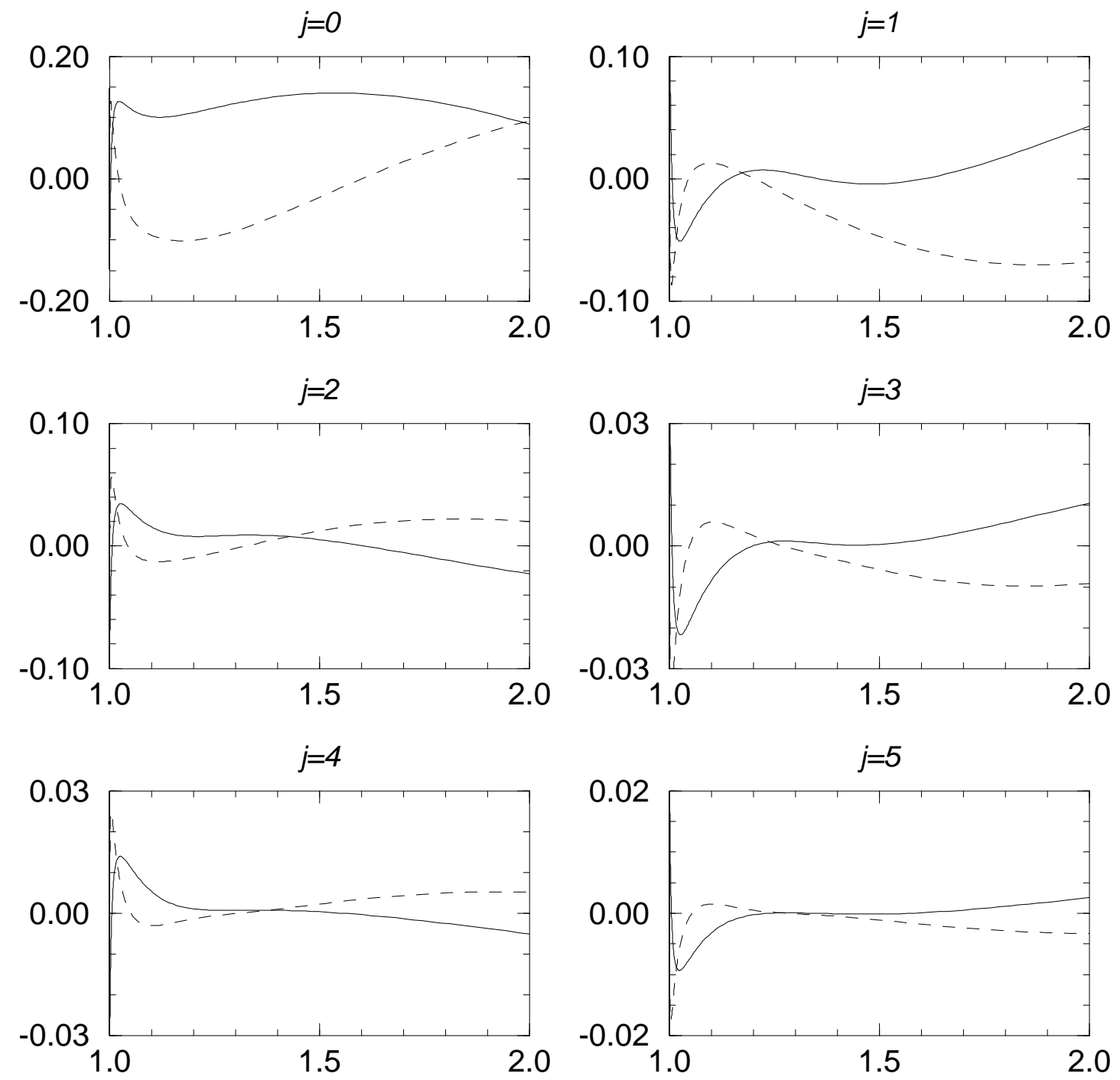

FIG. 3. Graph of Re $H(x) e^{-2 \Gamma \sqrt{1+(x / 2 M)^{2}}}$ (solid line) and $\operatorname{Im} H(x) e^{-2 \Gamma \sqrt{1+(x / 2 M)^{2}}}$ (dash line) vs $r / 2 M$. 

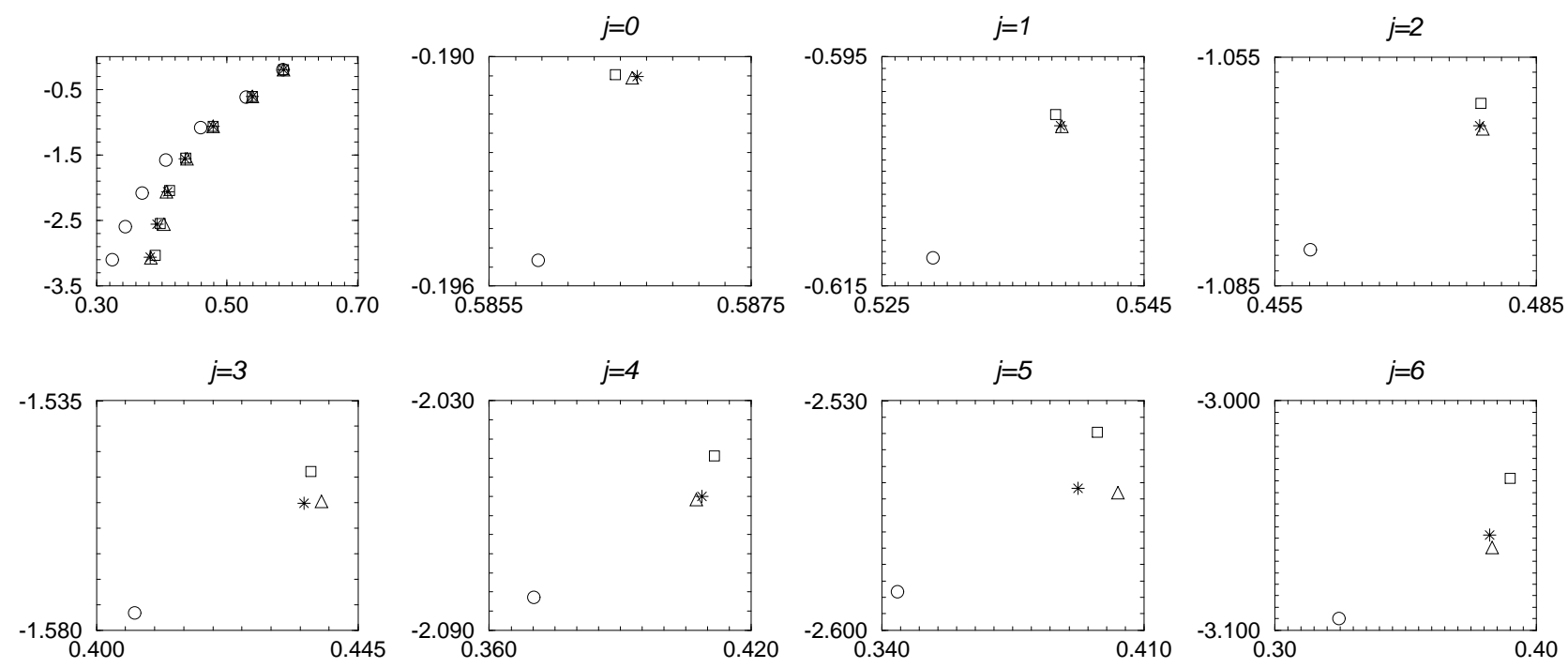

FIG. 4. The perturbed QNM spectrum of a black hole plus shell system for $l=1$ scalar wave with $\mu=0.02$ and $r_{s}=2.52 M_{a}$. The horizontal axis is $\operatorname{Re} 2 M_{o} \omega$ and the vertical axis is $\operatorname{Im} 2 M_{o} \omega$. The 0 th, 1st and 2 nd order perturbation result is indicated by circles, squares and triangles respectively. The exact numerical result is represented by stars. The diagram on the upper left shows the distribution of QNMs of the lowest damping modes (from $j=0$ to $j=6$ ). The other diagrams are the magnification of the region around each mode. 


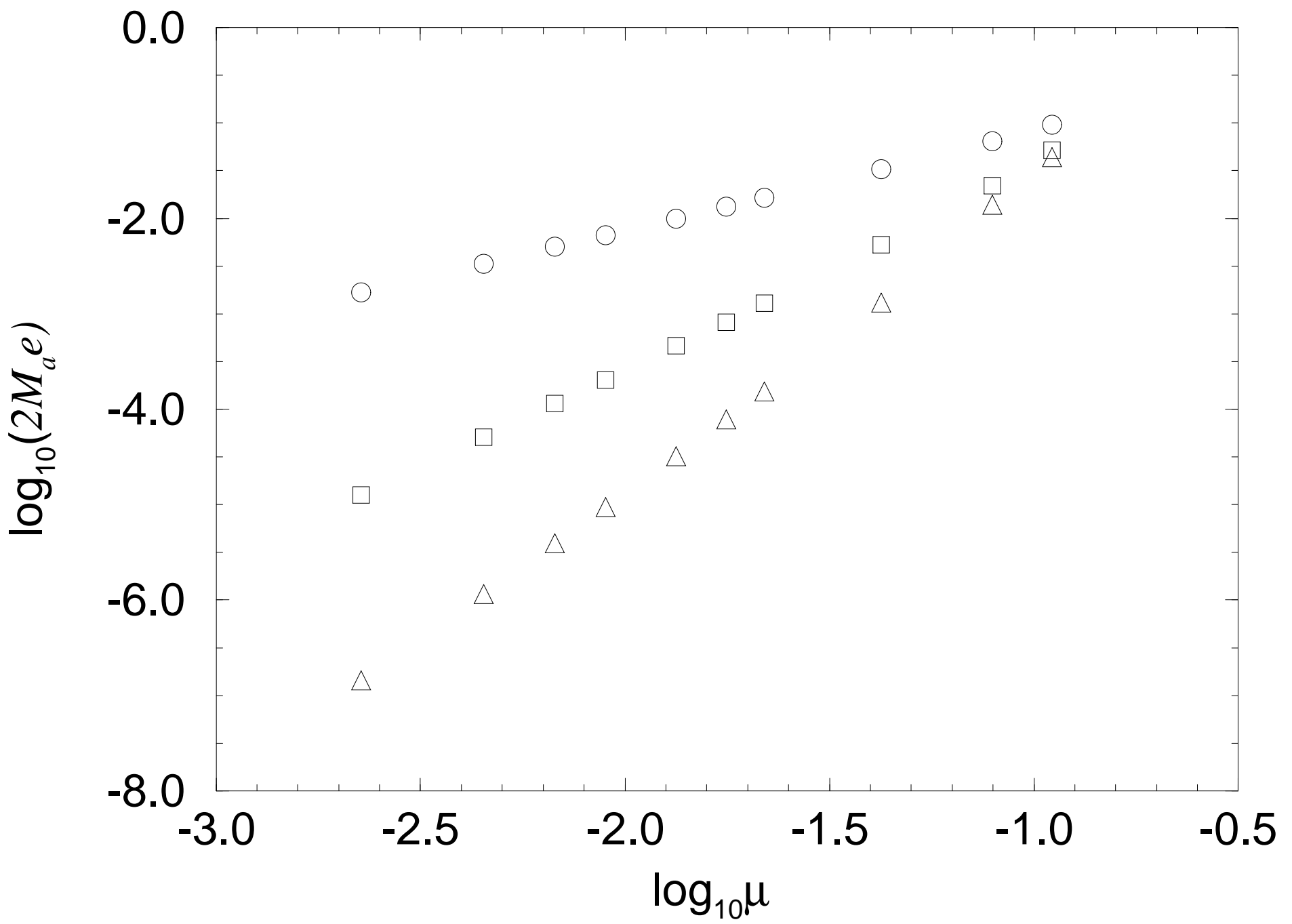

FIG. 5. The magnitude of the error in the frequencies of the 0th, 1st and 2nd order perturbation for $l=1, s=0, j=1$, and $r_{s}=2.52 M_{a}$. 


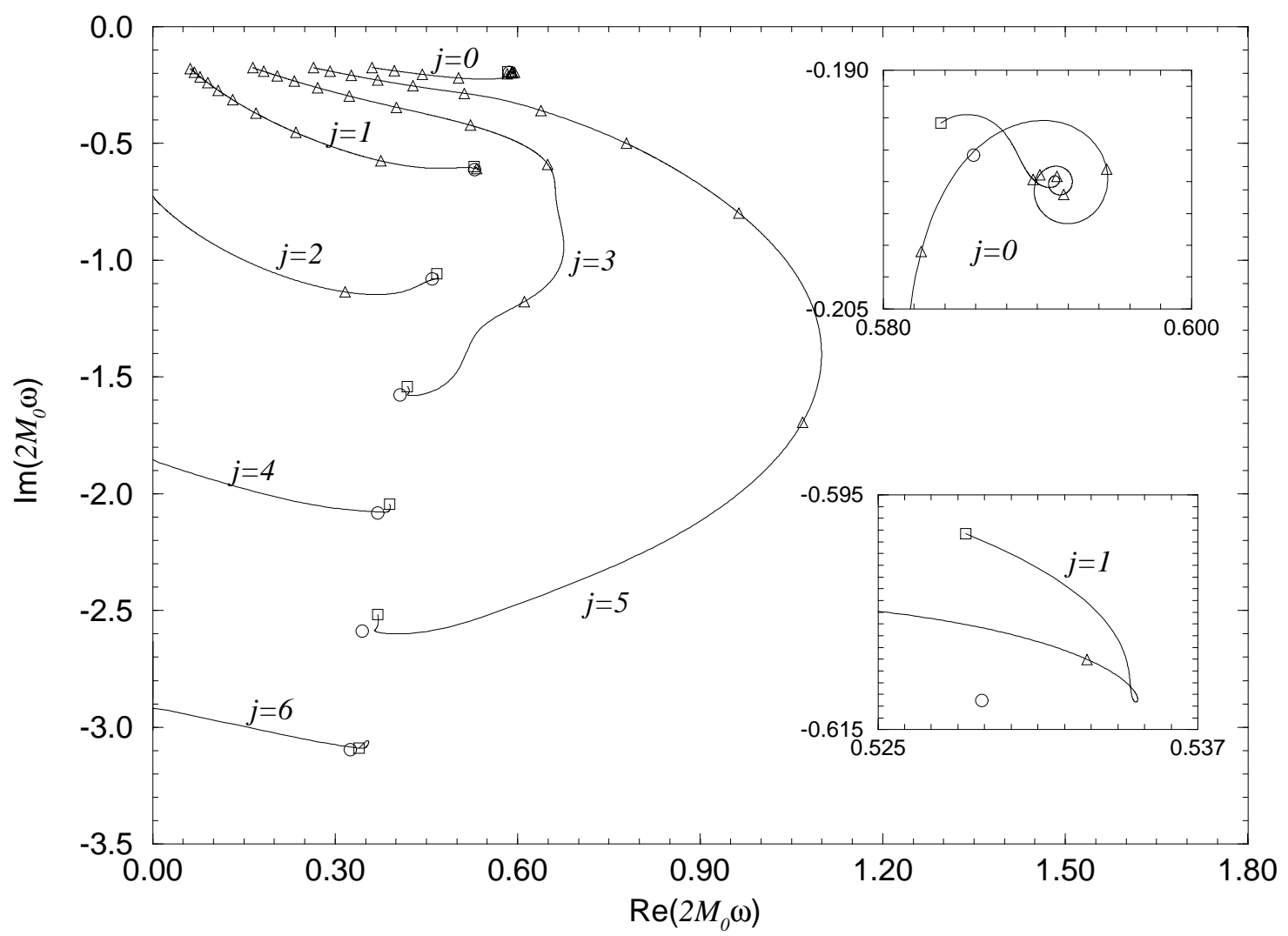

FIG. 6. The trajectory of the lowest QNMs of $l=1$ scalar waves for $\mu=0.01$ and $r_{s} / M_{a}$ varies from 2.26 to 60 based on exact numerical calculation. The circles are QNMs of a bare Schwarzschild black hole with mass $M_{o}$; the squares are the QNMs for $r_{s}=2.26 M_{a}$ (The dominant energy condition is violated when $r_{s}<2.26 M_{a}$ ); the triangles show the positions of QNMs at $r_{s} / M_{a}$ from 6 to 60 in intervals of 6 . The upper and lower inset show the regions near $j=0$ and $j=1$ in details respectively. We stopped the calculation when the QNMs approach the imaginary $\omega$ axis because it is difficult to compute it accurately in that region. Thus, some triangles appear "missing" for some higher damping QNMs because they have already moved to the imaginary $\omega$ axis. 


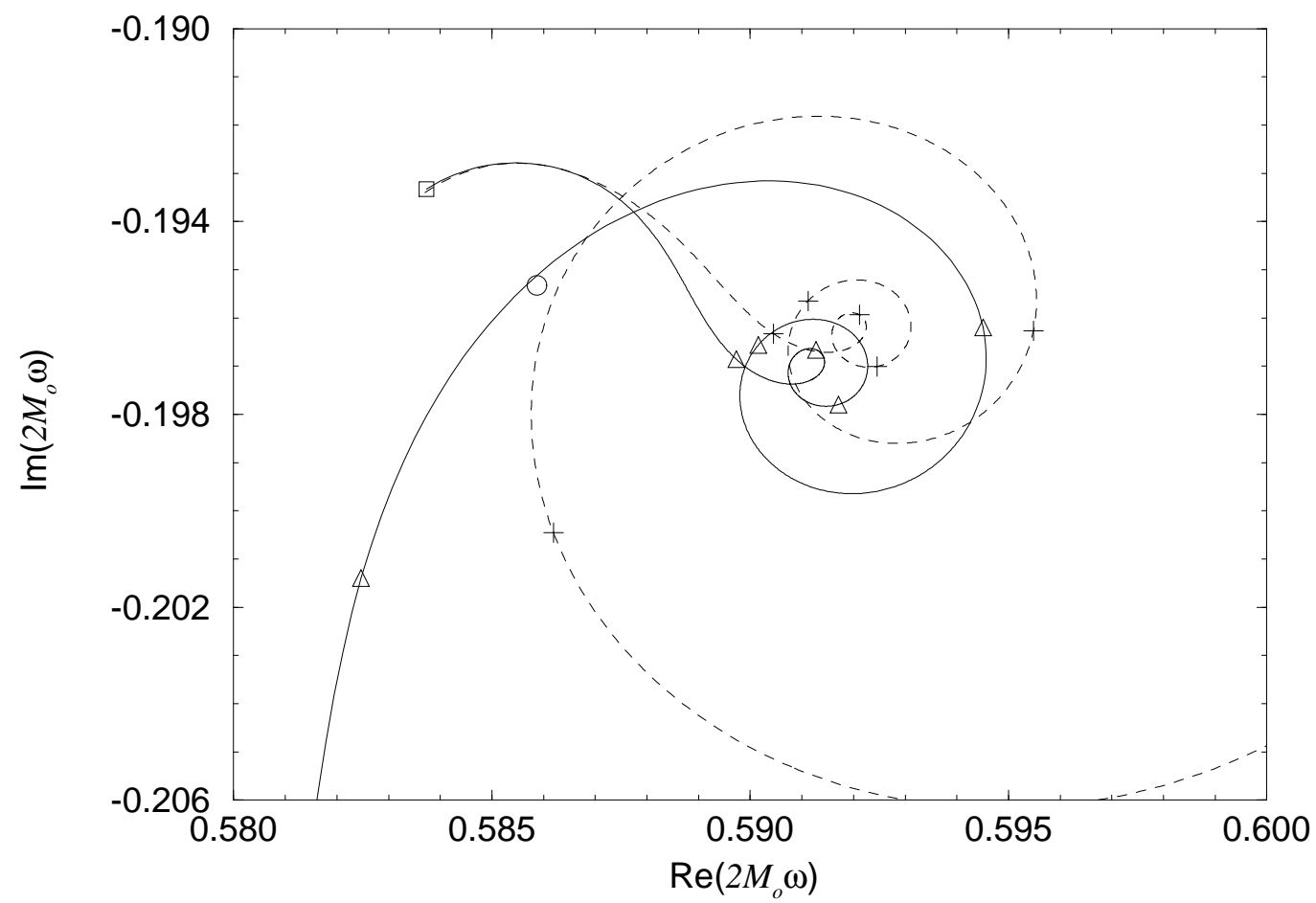

FIG. 7. Trajectory of $j=0$ mode based on exact (solid line) and first order (dash line) calculation. The zeroth order result is indicated by a circle and the square represents the mode for $r_{s}=2.26 M_{a}$. The triangles show the mode for the positions of the shell at $r_{s} / M_{a}$ from 6 to 36 in intervals of 6 for exact result, and the plus symbols show the first order result for the shell at the corresponding positions. 

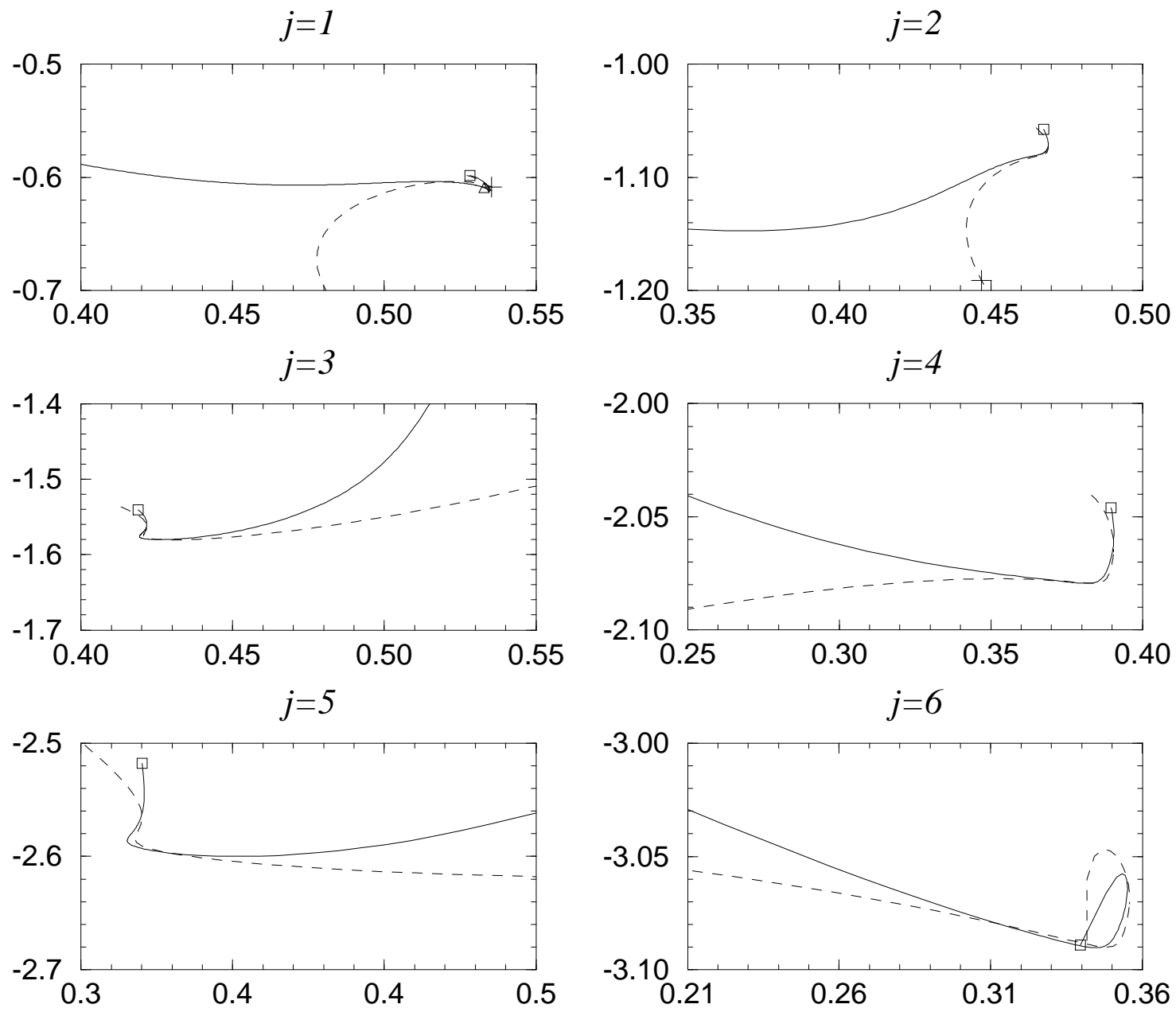

FIG. 8. Same as Fig. 7 but for $j=1,2, \cdots 6$. For these $j$, the perturbation breaks down before a spirl forms. 


\section{TABLES}

TABLE I. The normalizing factor $K /(2 M)$ (expressed as magnitude and phase) for the lowest QNMs of the Schwarzschild black hole with mass $M$.

\begin{tabular}{|l||c|r|c|c|c|r|}
\hline \multicolumn{1}{|c||}{} & \multicolumn{2}{c|}{$l=0, s=0$} & \multicolumn{2}{c|}{$l=1, s=0$} & \multicolumn{2}{c|}{$l=2, s=2$} \\
\hline$j$ & magnitude & phase & magnitude & phase & magnitude & phase \\
\hline 0 & 2.4461 & $36.5^{\circ}$ & 4.5155 & $36.7^{\circ}$ & 5.5397 & $18.7^{\circ}$ \\
1 & 193.24 & $18.4^{\circ}$ & 20.544 & $68.1^{\circ}$ & 6.9371 & $82.7^{\circ}$ \\
2 & 6.9935 & $12.9^{\circ}$ & 12.319 & $22.7^{\circ}$ & 22.770 & $-66.9^{\circ}$ \\
3 & 894.17 & $-10.2^{\circ}$ & 33.553 & $10.1^{\circ}$ & 9.4942 & $39.4^{\circ}$ \\
4 & 12.059 & $8.4^{\circ}$ & 18.945 & $20.5^{\circ}$ & 63.958 & $-89.7^{\circ}$ \\
5 & 2279.0 & $-10.4^{\circ}$ & 60.316 & $-14.2^{\circ}$ & 14.358 & $25.8^{\circ}$ \\
6 & 17.087 & $6.7^{\circ}$ & 25.087 & $26.6^{\circ}$ & 66.779 & $-100.9^{\circ}$ \\
\hline
\end{tabular}


TABLE II. The value of $H(x)$ (expressed as magnitude and phase) for several sample positions at $x=x_{1}\left(r=r_{1}\right)$, for $l=1$ scalar waves propagating on a Schwarzschild black hole.

\begin{tabular}{|r||l|l|l|r|l|r|l|r|r|r|}
\hline & \multicolumn{2}{|c|}{$x_{1} / 2 M=0$} & \multicolumn{2}{c|}{$x_{1} / 2 M=5$} & \multicolumn{2}{c|}{$x_{1} / 2 M=10$} & \multicolumn{2}{c|}{$r_{1} / 2 M=1.02$} & \multicolumn{2}{c|}{$r_{1} / 2 M=1.08$} \\
\hline$j$ & magnitude & phase & magnitude & phase & magnitude & phase & magnitude & phase & magnitude & phase \\
\hline 0 & $2.21 \times 10^{-1}$ & $-36.7^{\circ}$ & $9.18 \times 10^{-1}$ & $-122.2^{\circ}$ & $6.44 \times 10^{0}$ & $-147.1^{\circ}$ & $4.16 \times 10^{-1}$ & $4.8^{\circ}$ & $2.66 \times 10^{-1}$ & $-38.7^{\circ}$ \\
1 & $4.87 \times 10^{-2}$ & $-68.1^{\circ}$ & $5.23 \times 10^{1}$ & $109.8^{\circ}$ & $2.40 \times 10^{4}$ & $52.1^{\circ}$ & $2.69 \times 10^{1}$ & $-141.1^{\circ}$ & $2.14 \times 10^{-1}$ & $150.9^{\circ}$ \\
2 & $8.12 \times 10^{-2}$ & $-22.7^{\circ}$ & $2.88 \times 10^{3}$ & $-68.0^{\circ}$ & $1.28 \times 10^{8}$ & $-165.3^{\circ}$ & $3.14 \times 10^{1}$ & $39.1^{\circ}$ & $1.03 \times 10^{0}$ & $-28.5^{\circ}$ \\
3 & $2.98 \times 10^{-2}$ & $-10.1^{\circ}$ & $2.27 \times 10^{5}$ & $96.8^{\circ}$ & $1.60 \times 10^{12}$ & $-30.4^{\circ}$ & $4.20 \times 10^{2}$ & $-139.9^{\circ}$ & $3.32 \times 10^{0}$ & $156.4^{\circ}$ \\
4 & $5.28 \times 10^{-2}$ & $-20.5^{\circ}$ & $2.28 \times 10^{7}$ & $-99.7^{\circ}$ & $2.51 \times 10^{16}$ & $112.4^{\circ}$ & $6.00 \times 10^{3}$ & $40.2^{\circ}$ & $1.19 \times 10^{1}$ & $-19.2^{\circ}$ \\
5 & $1.66 \times 10^{-2}$ & $14.2^{\circ}$ & $2.61 \times 10^{9}$ & $65.9^{\circ}$ & $4.56 \times 10^{20}$ & $-97.0^{\circ}$ & $9.01 \times 10^{4}$ & $-140.2^{\circ}$ & $4.27 \times 10^{1}$ & $164.0^{\circ}$ \\
6 & $3.99 \times 10^{-2}$ & $-18.0^{\circ}$ & $3.22 \times 10^{11}$ & $-126.3^{\circ}$ & $8.91 \times 10^{24}$ & $59.6^{\circ}$ & $1.40 \times 10^{6}$ & $39.4^{\circ}$ & $1.57 \times 10^{2}$ & $13.6^{\circ}$ \\
\hline
\end{tabular}

